\title{
One-loop energy-momentum tensor in QED with electric-like background
}

\author{
S.P. Gavrilov*and D.M. Gitman ${ }^{\dagger}$
}

September 15, 2021

\begin{abstract}
We have obtained nonperturbative one-loop expressions for the mean energy-momentum tensor and current density of Dirac's field on a constant electric-like background. One of the goals of this calculation is to give a consistent description of back-reaction in such a theory. Two cases of initial states are considered: the vacuum state and the thermal equilibrium state. First, we perform calculations for the vacuum initial state. In the obtained expressions, we separate the contributions due to particle creation and vacuum polarization. The latter contributions are related to the Heisenberg-Euler Lagrangian. Then, we study the case of the thermal initial state. Here, we separate the contributions due to particle creation, vacuum polarization, and the contributions due to the work of the external field on the particles at the initial state. All these contributions are studied in detail, in different regimes of weak and strong fields and low and high temperatures. The obtained results allow us to establish restrictions on the electric field and its duration under which QED with a strong constant electric field is consistent. Under such restrictions, one can neglect the back-reaction of particles created by the electric field. Some of the obtained results generalize the calculations of Heisenberg-Euler for energy density to the case of arbitrary strong electric fields.
\end{abstract}

PACS numbers: 12.20.Ds,11.15.Tk,11.10.Wx

\section{Introduction}

It is well-known that quantum field theory (QFT) in external backgrounds is a good model for the study of quantum processes in cases when a part of a quantized field is strong enough to be treated as a classical one. Here, it is assumed that quantum processes under consideration do not affect significantly the classical field (back-reaction is supposed to be small) and the field is treated as an external one. Of course, it is well-understood that, in principle, back-reaction must be calculated, at any rate, to evaluate the limits of applicability of the obtained results. However, the latter problem often remains open due to its high complexity. From physical considerations, it is clear that back-reaction may be very strong, so it must be taken into account for external backgrounds that can create particles from vacuum (for theories with an unstable vacuum under the action of an external field) and produce actual work on particles. The effect of particle-creation from vacuum by an external field (vacuum instability in external fields) ranks among the most intriguing nonlinear phenomena in quantum theory. Its consideration is theoretically important, since it demands that one should go beyond the scope of perturbation theory, and its experimental observation would be able to verify the validity of a theory in the domain of superstrong fields. Concerning the history of the subject, the reader may refer to the books [1, 2, 3, 4, and to the articles [5] for recent computational developments. Typical problems examined in the models with an unstable vacuum are related to the calculation of the density of particles created from vacuum. In some cases, this allows one to make phenomenological conclusions about back-reaction; see, e.g., 6]. However, a complete description of back-reaction is related to the calculation of mean values for the current density and energy-momentum tensor (EMT) of the matter field. This problem, is, in fact, one of the goals of the present article. In this connection, we recall that Heisenberg and Euler calculated the mean value of energy density of Dirac's field in a constant electromagnetic background at zero temperature under the condition of weakness of the electric field $1 \mathbf{E},|\mathbf{E}| \ll E_{c}=M^{2} c^{3} / e \hbar \simeq 1,3 \cdot 10^{16} \mathrm{~V} / \mathrm{cm}$, see [7]. Having this energy density at hand, they constructed a one-loop effective Lagrangian $\mathcal{L}$. It turns out that this Lagrangian had been re-introduced by Schwinger [8] (for subsequent developments, see the review 9]) to define the vacuum-to-vacuum transition amplitude $c_{v}$ as follows:

$$
c_{v}=\exp \left(i \int d x \mathcal{L}\right)
$$

*Department of General and Experimental Physics, Herzen State Pedagogical University of Russia, Moyka emb. 48, 191186 St. Petersburg, Russia; e-mail: gavrilovsergeyp@yahoo.com

${ }^{\dagger}$ Institute of Physics, University of São Paulo, Brazil; e-mail: gitman@dfn.if.usp.br

${ }^{1} M$ is the electron mass and $e$ is the absolute value of the electron charge. 
without any restrictions on the external field. Schwinger demonstrated that the probability $P^{v}$ of a vacuum to remain a vacuum in a constant electric field is related to the imaginary part of $\mathcal{L}$ as follows:

$$
P^{v}=\left|c_{v}\right|^{2}=\exp \{-V T 2 \operatorname{Im} \mathcal{L}\} .
$$

Here, $T$ is the field duration, and $V$ is the volume of observation. Obviously, the effect can actually be observed as soon as the strength of the external field approaches the critical value $E_{c}$. Given this, there remains an open question of what is the form of the energy density, as well as the form of the entire EMT, in arbitrary constant fields. In the present article, we study the mean current density and EMT of a spinor field subject to the action of an electric-like external background, while paying a special attention to the initial state of thermal equilibrium. A more exact and detailed setting of the problem is given in the next section.

Aside from the principal importance of describing back-reaction for an understanding of the limits of applicability of QED in external fields, the calculations of the article are closely related to a wide class of problems (in the framework of quantum-field models in external fields) that recently attract considerable attention. Below, we present a brief review of these problems and of their importance, which may be regarded as an additional motivation for the present study.

Even though an actual possibility of creating superstrong fields under laboratory conditions does not exist at present, the $e^{+} e^{-}$-pair production from vacuum by a slowly varying external electric field is relevant to phenomenology with the advent of a new laser technology capable of accessing the true strongfield domain. It is widely discussed [10] at the SLAC and TESLA X-ray laser facilities. Such strong fields may be relevant in astrophysics, where characteristic values of electromagnetic and gravitational fields near black holes are enormous. The Coulomb barrier at the quark surface of a hot strange star may be a powerful source of $e^{+} e^{-}$-pairs, which are created in extremely strong constant electric fields (dozens of times higher that the critical field $E_{c}$ ) of the barrier and the flow away from the star [11. Such emission could be the main observational signature of quark stars. The electric field near a cosmic string can also be extremely strong $[12$.

Apart from pure QED problems, there exist some closely related QFT problems in which vacuum instability in various electric-like external backgrounds plays an important role, for example, phase transitions in non-Abelian theories, the problem of boundary conditions, or the influence of topology on vacuum, the problem of a consistent vacuum construction in QCD and GUT, multiple particle-creation in the context of heavy-ion collisions, etc. Recently, it has also been recognized that the presence of a background electric field must be taken into account in string theory constructions; see, e.g., [13] and references therein.

In the early 1970s, finite-temperature effects were recognized as very important in QFT, and then Dittrich 14 computed the one-loop effective potential of finite-temperature QED in the presence of a constant magnetic field, starting from the Schwinger relation (11). This was followed by an intense study of finite-temperature and finite-chemical-potential one-loop effects for fermions in a constant uniform magnetic field; see for a review 15, 16. This study was motivated by its possible applications to various areas of physics, including astrophysics, condensed matter, particle physics, etc., where strong electromagnetic fields may exist. The presence of an electric-like field implies a qualitative change (in comparison with a magnetic-like external field alone) in the character of processes involved, due to vacuum instability and the work of the field on the particles at the initial state.

In some of the above-mentioned examples with an electric-like external field, the initial state is in thermal equilibrium. In the chromoelectric flux-tube model [17, the back-reaction of created pairs induces a gluon mean field and plasma oscillations (see [6] and references therein). Various problems of cosmological QCD phase-transitions and dark matter formation are discussed on the basis of the chromoelectric flux tube model (see, for example, [18, and references therein). It appears that for a calculation of particle creation in this model one needs to apply the general formalism of QFT for pair-production at finite temperatures and at zero temperature, both from vacuum and from many-particle states (see the corresponding physical reasons in [19, 20, 21]). A consideration of various time scales in heavy-ion collisions shows that the stabilization time (a time interval during which the characteristic asymptotic form for differential mean numbers of particles created by a constant chromoelectric field from vacuum is achieved) is far less than the period of plasma and mean-field oscillations. Then, the approximation of a strong quasiconstant chromoelectric field can be used in the treatment of such collisions during a period when the partons produced can be regarded as weakly coupled, due to the property of asymptotic freedom in QCD. It may also be reasonable to neglect dynamical back-reaction effects [22. In the case of a strange star [11, it has been argued that there is a macroscopic time interval during which local thermal equilibrium is achieved, but the strong electric field has not yet been depleted, due to $e^{+} e^{-}$-pair production. By this reason, there has been a considerable interest in establishing a relevant formalism for finite-temperature QED with an unstable vacuum. An adequate techniques for such a case has been proposed in 23]. The special case of thermally-influenced pair production in a constant electric field has been studied at the one-loop level in $19,22,24,25,26$. The results of $22,24,25$, obtained by various methods in the framework of the generalized Furry representation, are in mutual agreement. The authors of 19, 26, have arrived at different results, being, at the same time, in contradiction with themselves. 
We believe that the difficulties [19, 26] are related to some attempts to generalize relation (1) to nonzero temperatures; see Discussion and Summary.

The paper is organized as follows. In Section 2, we describe the setting of the problem and recall some necessary details of the non-perturbative techniques that we use in our calculations. In Section 3, we calculate the current density and EMT for the vacuum initial state. In the obtained expressions, we separate the contributions due to particle creation and vacuum polarization. The latter contributions are related to the Heisenberg-Euler Lagrangian. In Section 4, we calculate the current density and EMT for the thermal initial state. In the obtained expressions, we separate the contributions due to particlecreation, vacuum polarization, and the contributions that appear due to the work of the external field on the particles at the initial state. All these contributions are studied in detail in different regimes, limits of weak and strong fields and low and high temperatures. We have established restrictions on the electric field and its duration under which QED with a strong constant electric field is consistent. Within these restrictions, one can neglect the back-reaction of particles created by the electric field. In the last section, we summarize and discuss the main results. Some necessary but cumbersome calculations are placed in the Appendix.

In what follows, we use the relativistic units $\hbar=c=1$ in which the fine structure constant is $\alpha=e^{2} / c \hbar=e^{2}$.

\section{The setting of the problem}

We consider a quantized spinor (Dirac) field $\psi(x), x=\left(x^{0}=t, \mathbf{x}\right)$, in an external electromagnetic background specified by a quasiconstant electric field and a constant magnetic field. The field strength $F_{\mu \nu}$ has nonzero invariants. In this case, we can always choose a reference frame in which the electric and magnetic fields are parallel and directed along the $x^{3}$-axis:

$$
F_{\mu \nu}=F_{\mu \nu}^{E}+F_{\mu \nu}^{B}, \quad F_{\mu \nu}^{E}=E\left(\delta_{\mu}^{0} \delta_{\nu}^{3}-\delta_{\mu}^{3} \delta_{\nu}^{0}\right), \quad F_{\mu \nu}^{B}=B\left(\delta_{\mu}^{2} \delta_{\nu}^{1}-\delta_{\mu}^{1} \delta_{\nu}^{2}\right) .
$$

Our aim is to study the mean values of electric current and EMT in states that have evolved from vacuum and thermal equilibrium. It is well-known that a uniform constant electric field acting during an infinite time creates an infinite number density of pairs from vacuum and produces infinite work on particles. There arise other divergences in the course of QED calculations related to this phenomenon. This is why one needs a regularization to deal with these divergences. One type of such a regularization is the following, namely, we consider, from the beginning, a uniform electric field, which efficiently acts only during a sufficiently large but finite time $T$. Such a regularization has been used in our works [3, 27, 28]. In these works, we studied particle-creation effects in the so-called $T$-constant field, being a uniform electric field, constant (and nonzero) within a time interval $T$, and zero outside this interva[2]. A similar regularization is used in the present article: we choose the $T$-constant field to switch on at the instant $t_{1}=-T / 2$ and to switch off at the instant $t_{2}=T / 2$. The results of our calculations will be presented in a form in which contributions of different nature are separated. These are contributions due to vacuum polarization, particle-creation from vacuum, and the contributions due to the work of the external field on the particles at the initial state. Some of these contributions have constant parts, as well as parts depending on the time interval $t-t_{1}$ that passes since the instant the electric field turns on. Among the contributions depending on $t-t_{1}$, there exist contributions that can increase and decrease as the interval $t-t_{1}$ increases. Having the general expressions at hand, we analyze their dependence on the magnitude $E$ of electric field and the time interval $t-t_{1}$, as well as their behavior at low and high temperatures. In the case of a strong electric field, when among the parts depending on $t-t_{1}$ the leading ones are the contributions due to particle-creation, we are interested in the divergent parts related to a large $t-t_{1}$. In the latter parts, we retain only the contributions leading in $t-t_{1}$. In particular, we analyze the dynamics of mean-energy growth due to particle creation from vacuum and due to the work of electric field on real particles. Making a comparison of this dynamics with the energy density of the external electric field, we can establish the limits of a correct applicability of the concepts of a constant external field. In our calculations, we use the technics and general results outlined below in this section with the corresponding citations.

We assume the initial state to be a state of free $i n$-particles in thermal equilibrium at a given temperature $\theta$. In such a model, we are going to calculate the mean values of electric current and the EMT of the Dirac field. In the general case, the background under consideration is intense, time-dependent and

\footnotetext{
${ }^{2}$ The physical interpretation in the case of a field that violates the stability of vacuum (for instance, a constant electric field) is extremely involved when the field is given by a space-dependent potential which does not disappear asymptotically. This happens because the state space of strong-field QFT is quite different from the state space of standard scattering theory, in which such a gauge for an external field is appropriate. In the general case of a space-dependent potential, one cannot use the standard definition of vacuum in QFT because such a state is well-defined only on a space-like hypersurface. It is then generally unclear when a strong electric field is given by a space-dependent potential, what particles and antiparticles are, what the vacuum state is, and what is the connection of the definitions being applied with the standard QFT approach. In this case, instead of the standard evolution in time, one has to use the rather obscure "evolution in space".
} 
violates the vacuum stability. Such a background must be considered nonperturbatively. In doing this, we follow the formulation proposed in 29 . Our calculations can be treated as a one-loop approximation within the method of complete QED, developed in [3, 30] and [23, 22. Namely, this method provides a technique of calculating the mean values for systems with unstable vacuum, which is based on the generalized Furry picture for systems in strong external backgrounds (see [29, 3]); this technique may be interpreted as a generalization of the Schwinger-Keldysh technique ([31]; see also the review [32]) on systems with unstable vacuum.

It is assumed that in the Heisenberg picture (for notation, see [3, 22]) there exists a set of creation and annihilation operators $a_{n}^{\dagger}(i n), a_{n}$ (in) of $i n$-particles (electrons), and analogous operators $b_{n}^{\dagger}(i n)$, $b_{n}(i n)$ of $i n$-antiparticles (positrons), with the corresponding $i n$-vacuum $|0, i n\rangle$, and a set of creation and annihilation operators $a_{n}^{\dagger}($ out $), a_{n}$ (out), of out-electrons and similar operators $b_{n}^{\dagger}($ out $), b_{n}$ (out) of out-positrons, with the corresponding out-vacuum $\mid 0$, out $\rangle$. By $n$, we denote a complete set of possible quantum numbers. The $i n$ - and out-operators obey the canonical anticommutation relations

$$
\left[a_{n}(\text { in }), a_{m}^{\dagger}(\text { in })\right]_{+}=\left[a_{n}(\text { out }), a_{m}^{\dagger}(\text { out })\right]_{+}=\left[b_{n}(\text { in }), b_{m}^{\dagger}(\text { in })\right]_{+}=\left[b_{n}(\text { out }), b_{m}^{\dagger}(\text { out })\right]_{+}=\delta_{n m} .
$$

The $i n$-particles $(\zeta=+$ for electrons and $\zeta=-$ for positrons) are associated with a complete set of solutions $\left\{{ }_{\zeta} \psi_{n}(x)\right\}$ (we call it an in-set in what follows) of the Dirac equation with the external electromagnetic field under consideration with the asymptotics $\left\{{ }_{\zeta} \psi_{n}\left(t_{1}, \mathbf{x}\right)\right\}$ at the initial time-instant $t_{1}$. The latter functions are eigenvectors of the one-particle Dirac Hamiltonian $\mathcal{H}(t)$ (with external field under consideration; see its exact definition in the next section) at $t=t_{1}$,

$$
\mathcal{H}\left(t_{1}\right)_{\zeta} \psi_{n}\left(t_{1}, \mathbf{x}\right)=\zeta \varepsilon_{n}^{(\zeta)}{ }_{\zeta} \psi_{n}\left(t_{1}, \mathbf{x}\right), \mathcal{H}(t)=\gamma^{0}([M+\gamma(i \boldsymbol{\nabla}-q \mathbf{A}(t, \mathbf{x}))]),
$$

where $\gamma^{\mu}=\left(\gamma^{0}, \gamma\right)$ are Dirac's gamma-matrices; $q$ is the particle charge (for an electron $q=-e$ ); $\mathbf{A}$ is the vector potential of the external electromagnetic field ( $A_{0}$ is zero in the case under consideration); $\varepsilon_{n}^{(\zeta)}$ are the energies of $i n$-particles in a state specified by a complete set of quantum numbers $n$, and $\varepsilon_{n}^{( \pm)}>0$. The out-particles are associated with a complete out-set of solutions $\left\{{ }^{\zeta} \psi_{n}(x)\right\}$ of the Dirac equation with the asymptotics ${ }^{\zeta} \psi_{n}\left(t_{2}, \mathbf{x}\right)$ at $t_{2}$ being eigenvectors of the one-particle Dirac Hamiltonian at $t_{2}$, namely,

$$
\mathcal{H}\left(t_{2}\right)^{\zeta} \psi_{n}\left(t_{2}, \mathbf{x}\right)=\zeta \tilde{\varepsilon}_{n}^{(\zeta) \zeta} \psi_{n}\left(t_{2}, \mathbf{x}\right),
$$

where $\tilde{\varepsilon}_{n}^{( \pm)}$are the energies of out-particles in a state specified by a complete set of quantum numbers $n$, and $\tilde{\varepsilon}_{n}^{( \pm)}>0$.

The out-set can be decomposed in the in-set as follows:

$$
{ }^{\zeta} \psi(x)={ }_{+} \psi(x) G\left(\left.{ }_{+}\right|^{\zeta}\right)+{ }_{-} \psi(x) G\left(\left.{ }_{-}\right|^{\zeta}\right),
$$

where the decomposition coefficients $G\left(\left.{ }_{\zeta}\right|^{\zeta^{\prime}}\right)$ are expressed via the inner products of these sets. These coefficients obey unitary conditions that follow from normalization conditions for the solutions.

The Hamiltonian $H(t)$ of the quantized Dirac field is time-dependent due to the external field. It is diagonalized (and has a canonical form) in terms of the first set at the initial time instant, and is diagonalized (and has a canonical form) in terms of the second set at the final time instant. For example,

$$
H\left(t_{1}\right)=\sum_{n}\left[\varepsilon_{n}^{(+)} a_{n}^{\dagger}(\text { in }) a_{n}(\text { in })+\varepsilon_{n}^{(-)} b_{n}^{\dagger}(\text { in }) b_{n}(\text { in })\right] .
$$

Correspondingly, the initial vacuum is defined by $a_{n}(i n)|0, i n\rangle=b_{n}(i n)|0, i n\rangle=0$ for every $n$.

All the information about the processes of particle-creation, annihilation, and scattering is contained in the elementary probability amplitudes

$$
\begin{aligned}
& w(+\mid+)_{m n}=c_{v}^{-1}<0, \text { out } \mid a_{m}(\text { out }) a_{n}^{\dagger}(\text { in }) \mid 0, \text { in }>=G^{-1}\left(\left.{ }_{+}\right|^{+}\right)_{m n}, \\
& w(-\mid-)_{n m}=c_{v}^{-1}<0, \text { out } \mid b_{m}(\text { out }) b_{n}^{\dagger}(\text { in }) \mid 0, \text { in }>=\left[G^{-1}\left(\left.{ }_{-}\right|^{-}\right)\right]_{n m}^{\dagger}, \\
& w(0 \mid-+)_{n m}=c_{v}^{-1}<0, \text { out } \mid b_{n}^{\dagger}(\text { in }) a_{m}^{\dagger}(\text { in }) \mid 0, \text { in }>=-\left[G\left(+\left.\right|^{-}\right) G^{-1}\left(\left.{ }_{-}\right|^{-}\right)\right]_{n m}^{\dagger}, \\
& w(+-\mid 0)_{m n}=c_{v}^{-1}<0, \text { out } \mid a_{m}(\text { out }) b_{n}(\text { out }) \mid 0, \text { in }>=\left[G^{-1}\left(\left.{ }_{+}\right|^{+}\right) G\left(\left.{ }_{+}\right|^{-}\right)\right]_{m n}, \\
& \left.c_{v}=\langle 0, \text { out }| 0, \text { in }\right\rangle,
\end{aligned}
$$

where $c_{v}$ is the vacuum-to-vacuum transition amplitude.

The sets of in and out-operators are related to each other by a linear canonical transformation (sometimes called the Bogolyubov transformation). It has been demonstrated that in the general case such a relation has the form (see [29])

$$
\left.\left.V\left(a^{\dagger}(\text { out }), a(\text { out }), b^{\dagger}(\text { out }), b(\text { out })\right) V^{\dagger}=\left(a^{\dagger}(\text { in }), a(\text { in }), b^{\dagger}(\text { in }), b(\text { in })\right), \mid 0, \text { in }\right\rangle=V \mid 0, \text { out }\right\rangle,
$$


where the unitary operator $V$ has the form 3

$$
\begin{aligned}
V= & \exp \left\{-a^{\dagger}(\text { out }) w(+-\mid 0) b^{\dagger}(\text { out })\right\} \exp \left\{-b(\text { out }) \ln w(-\mid-) b^{\dagger}(\text { out })\right\} \\
& \times \exp \left\{a^{\dagger}(\text { out }) \ln w(+\mid+) a(\text { out })\right\} \exp \{-b(\text { out }) w(0 \mid-+) a(\text { out })\},
\end{aligned}
$$

and

$$
c_{v}=\langle 0, \text { out }|V| 0, \text { out }\rangle=\exp \{-\operatorname{Tr} \ln w(-\mid-)\} .
$$

Note that the exact formula (12) holds true for an arbitrary external field. In 33, has been demonstrated that in the case of a constant electric-like field the Schwinger formula (1) leads to the same result.

As our final purpose, we are going to analyze the following mean values:

$$
\left\langle j_{\mu}(t)\right\rangle=\operatorname{Tr}\left[\rho_{i n} j_{\mu}\right],\left\langle T_{\mu \nu}(t)\right\rangle=\operatorname{Tr}\left[\rho_{i n} T_{\mu \nu}\right],
$$

where $\rho_{i n}$ is a density operator of the initial state in the Heisenberg picture; the operators of current density $j_{\mu}$, and energy-momentum tensor $T_{\mu \nu}$ have the form

$$
\begin{aligned}
& j_{\mu}=\frac{q}{2}\left[\bar{\psi}(x), \gamma_{\mu} \psi(x)\right], T_{\mu \nu}=\frac{1}{2}\left(T_{\mu \nu}^{c a n}+T_{\nu \mu}^{c a n}\right), \\
& T_{\mu \nu}^{c a n}=\frac{1}{4}\left\{\left[\bar{\psi}(x), \gamma_{\mu} P_{\nu} \psi(x)\right]+\left[P_{\nu}^{*} \bar{\psi}(x), \gamma_{\mu} \psi(x)\right]\right\} .
\end{aligned}
$$

Here, $P_{\mu}=i \partial_{\mu}-q A_{\mu}(x)$ and $\psi(x)$ are Dirac field operators in the Heisenberg representation that obey the Dirac equation with the external field $A_{\mu}(x)$.

In the next section, we calculate and analyze the mean values (13) for the initial vacuum state. We denote them as follows:

$$
\left\langle j_{\mu}(t)\right\rangle^{0}=\left\langle 0, i n\left|j_{\mu}\right| 0, i n\right\rangle,\left\langle T_{\mu \nu}(t)\right\rangle^{0}=\left\langle 0, i n\left|T_{\mu \nu}\right| 0, i n\right\rangle .
$$

Then, we analyze the more complicated case (13), in which the initial state of the system under consideration is prepared as an equilibrium state of noninteracting in-particles at temperature $\theta$ with the chemical potentials $\mu^{(\zeta)}$, and is characterized by the density operator $\rho_{i n}$ in the Heisenberg picture,

$$
\rho_{\text {in }}=Z^{-1} \exp \left\{\beta\left(\sum_{\zeta= \pm} \mu^{(\zeta)} N^{(\zeta)}-H\left(t_{1}\right)\right)\right\}, \operatorname{Tr} \rho_{\text {in }}=1,
$$

where $Z$ is a normalization constant, $\beta=\theta^{-1}$, and $N^{(\zeta)}$ are the operators of $i n$-paricles numbers,

$$
N^{(+)}=\sum_{n} a_{n}^{\dagger}(\text { in }) a_{n}(\text { in }), N^{(-)}=\sum_{n} b_{n}^{\dagger}(\text { in }) b_{n}(\text { in }) .
$$

In our calculations, we also need the matrix elements

$$
\begin{gathered}
\left\langle j_{\mu}(t)\right\rangle^{c}=\left\langle 0, \text { out }\left|j_{\mu}\right| 0, \text { in }\right\rangle c_{v}^{-1},\left\langle T_{\mu \nu}(t)\right\rangle^{c}=\left\langle 0, \text { out }\left|T_{\mu \nu}\right| 0, \text { in }\right\rangle c_{v}^{-1}, \\
\left\langle j_{\mu}(t)\right\rangle_{\text {out }}^{0}=\left\langle 0, \text { out }\left|j_{\mu}\right| 0, \text { out }\right\rangle,\left\langle T_{\mu \nu}(t)\right\rangle_{\text {out }}^{0}=\left\langle 0, \text { out }\left|T_{\mu \nu}\right| 0, \text { out }\right\rangle .
\end{gathered}
$$

The matrix elements (13), (14), and (16) can be expressed via relevant singular functions of Dirac's fields, as follows:

$$
\begin{aligned}
& i \operatorname{Tr}\left\{\rho_{i n} T \psi(x) \bar{\psi}\left(x^{\prime}\right)\right\}=S_{i n}^{c}\left(x, x^{\prime}\right)+S^{\theta}\left(x, x^{\prime}\right), \\
& i \operatorname{Tr}\left\{\rho_{i n} \bar{\psi}\left(x^{\prime}\right) \psi(x)\right\}=S_{i n}^{+}\left(x, x^{\prime}\right)-S^{\theta}\left(x, x^{\prime}\right), \\
& i \operatorname{Tr}\left\{\rho_{i n} \psi(x) \bar{\psi}\left(x^{\prime}\right)\right\}=S_{i n}^{-}\left(x, x^{\prime}\right)+S^{\theta}\left(x, x^{\prime}\right), \\
& S_{i n}^{c}\left(x, x^{\prime}\right)=\theta\left(x_{0}-x_{0}^{\prime}\right) S_{i n}^{-}\left(x, x^{\prime}\right)-\theta\left(x_{0}^{\prime}-x_{0}\right) S_{i n}^{+}\left(x, x^{\prime}\right),
\end{aligned}
$$

where $S_{i n}^{(\ldots)}$ are singular functions at zero temperature,

$$
\begin{aligned}
S_{i n}^{c}\left(x, x^{\prime}\right) & =i\left\langle 0, i n\left|T \psi(x) \bar{\psi}\left(x^{\prime}\right)\right| 0, i n\right\rangle, \\
S_{i n}^{-}\left(x, x^{\prime}\right) & =i\left\langle 0, i n\left|\psi(x) \bar{\psi}\left(x^{\prime}\right)\right| 0, i n\right\rangle, \\
S_{i n}^{+}\left(x, x^{\prime}\right) & =i\left\langle 0, i n\left|\bar{\psi}\left(x^{\prime}\right) \psi(x)\right| 0, i n\right\rangle .
\end{aligned}
$$

\footnotetext{
${ }^{3}$ Here and elsewhere, we use condenced notations, for example,

$$
b w(0 \mid-+) a=\sum_{n, m} b_{n} w(0 \mid-+)_{n m} a_{m} .
$$
}


All the singular functions can be expressed in terms of the $i n$-set of solutions as follows:

$$
\begin{aligned}
& S_{i n}^{\mp}\left(x, x^{\prime}\right)=i \sum_{n}{ }_{ \pm} \psi_{n}(x)_{ \pm} \bar{\psi}_{n}\left(x^{\prime}\right), \\
& S^{\theta}\left(x, x^{\prime}\right)=\tilde{S}_{\theta}^{-}\left(x, x^{\prime}\right)-\tilde{S}_{\theta}^{+}\left(x, x^{\prime}\right) \\
& \tilde{S}_{\theta}^{\mp}\left(x, x^{\prime}\right)=-i \sum_{n}{ }_{ \pm} \psi_{n}(x)_{ \pm} \bar{\psi}_{n}\left(x^{\prime}\right) N_{n}^{( \pm)}(i n),
\end{aligned}
$$

where

$$
N_{n}^{(\zeta)}(i n)=\left[\exp \left\{\beta\left(\varepsilon_{n}^{(\zeta)}-\mu^{(\zeta)}\right)\right\}+1\right]^{-1} .
$$

It is important to emphasize that, in the general case, $S_{i n}^{c}$ is different from the causal Green function,

$$
S^{c}\left(x, x^{\prime}\right)=i\left\langle 0, \text { out }\left|T \psi(x) \bar{\psi}\left(x^{\prime}\right)\right| 0, \text { in }\right\rangle c_{v}^{-1} .
$$

From (21), it follows that

$$
\begin{aligned}
& S^{c}\left(x, x^{\prime}\right)=\theta\left(x_{0}-x_{0}^{\prime}\right) S^{-}\left(x, x^{\prime}\right)-\theta\left(x_{0}^{\prime}-x_{0}\right) S^{+}\left(x, x^{\prime}\right), \\
& S^{-}\left(x, x^{\prime}\right)=i \sum_{n, m}{ }^{+} \psi_{n}(x) G\left(+\left.\right|^{+}\right)_{n m}^{-1}+\bar{\psi}_{m}\left(x^{\prime}\right) \\
& S^{+}\left(x, x^{\prime}\right)=i \sum_{n, m}-\psi_{n}(x)\left[G\left(-\left.\right|^{-}\right)^{-1}\right]_{n m}^{\dagger}-\bar{\psi}_{m}\left(x^{\prime}\right),
\end{aligned}
$$

see [29]. Then the difference $S^{p}\left(x, x^{\prime}\right)=S_{i n}^{c}\left(x, x^{\prime}\right)-S^{c}\left(x, x^{\prime}\right)$ has the form

$$
S^{p}\left(x, x^{\prime}\right)=i \sum_{n m}{ }_{-} \psi_{n}(x)\left[G\left(+\left._{+}\right|^{-}\right) G\left(\left.{ }_{-}\right|^{-}\right)^{-1}\right]_{n m}^{\dagger}+\bar{\psi}_{m}\left(x^{\prime}\right) .
$$

This function vanishes in the case of a stable vacuum, since it contains the coefficients $G\left(\left.{ }_{+}\right|^{-}\right)$related to the mean number of created particles.

Another kind of Green's function that will be used later on is given by

$$
S_{\text {out }}^{c}\left(x, x^{\prime}\right)=i\left\langle 0, \text { out }\left|T \psi(x) \bar{\psi}\left(x^{\prime}\right)\right| 0, \text { out }\right\rangle .
$$

It is related to $S^{c}\left(x, x^{\prime}\right)$ as follows:

$$
\begin{aligned}
& S_{\text {out }}^{c}\left(x, x^{\prime}\right)=S^{c}\left(x, x^{\prime}\right)+S^{\bar{p}}\left(x, x^{\prime}\right), \\
& S^{\bar{p}}\left(x, x^{\prime}\right)=-i \sum_{n m}{ }^{+} \psi_{n}(x)\left[G\left(\left.{ }_{+}\right|^{+}\right)^{-1} G\left(\left.{ }_{+}\right|^{-}\right)\right]_{n m}-\bar{\psi}_{m}\left(x^{\prime}\right) .
\end{aligned}
$$

We can see that there exist relations between the mean values (13), (14) and matrix elements (16), namely,

$$
\begin{array}{ccc}
\left\langle j_{\mu}(t)\right\rangle= & \left\langle j_{\mu}(t)\right\rangle^{0}+\left\langle j_{\mu}(t)\right\rangle^{\theta}, \quad\left\langle T_{\mu \nu}(t)\right\rangle=\left\langle T_{\mu \nu}(t)\right\rangle^{0}+\left\langle T_{\mu \nu}(t)\right\rangle^{\theta}, \\
\left\langle j_{\mu}(t)\right\rangle^{0}= & \left\langle j_{\mu}(t)\right\rangle^{c}+\left\langle j_{\mu}(t)\right\rangle^{p}, \quad\left\langle T_{\mu \nu}(t)\right\rangle^{0}=\left\langle T_{\mu \nu}(t)\right\rangle^{c}+\left\langle T_{\mu \nu}(t)\right\rangle^{p}, \\
\left\langle j_{\mu}(t)\right\rangle_{\text {out }}^{0}= & \left\langle j_{\mu}(t)\right\rangle^{c}+\left\langle j_{\mu}(t)\right\rangle^{\bar{p}}, \quad\left\langle T_{\mu \nu}(t)\right\rangle_{\text {out }}^{0}=\left\langle T_{\mu \nu}(t)\right\rangle^{c}+\left\langle T_{\mu \nu}(t)\right\rangle^{\bar{p}} .
\end{array}
$$

These relations involve the quantities

$$
\begin{aligned}
& \left\langle j_{\mu}(t)\right\rangle^{c, p, \bar{p}, \theta}=\left.i q \operatorname{tr}\left[\gamma_{\mu} S^{c, p, \bar{p}, \theta}\left(x, x^{\prime}\right)\right]\right|_{x=x^{\prime}}, \\
& \left\langle T_{\mu \nu}(t)\right\rangle^{c, p, \bar{p}, \theta}=\left.i \operatorname{tr}\left[A_{\mu \nu} S^{c, p, \bar{p}, \theta}\left(x, x^{\prime}\right)\right]\right|_{x=x^{\prime}}, \\
& A_{\mu \nu}=1 / 4\left[\gamma_{\mu}\left(P_{\nu}+P_{\nu}^{\prime *}\right)+\gamma_{\nu}\left(P_{\mu}+P_{\mu}^{\prime *}\right)\right], \\
& P_{\mu}^{\prime *}=-i \frac{\partial}{\partial x^{\prime \mu}}-q A_{\mu}\left(x^{\prime}\right),
\end{aligned}
$$

where $\operatorname{tr}[\cdots]$ is the trace in the space of $4 \times 4$ matrices, and the equality $x=x^{\prime}$ is understood as follows:

$$
\left.\operatorname{tr}\left[\cdots\left(x, x^{\prime}\right)\right]\right|_{x=x^{\prime}}=\left.\frac{1}{2}\left[\lim _{t \rightarrow t^{\prime}-0} \operatorname{tr}\left[\cdots\left(x, x^{\prime}\right)\right]+\lim _{t \rightarrow t^{\prime}+0} \operatorname{tr}\left[\cdots\left(x, x^{\prime}\right)\right]\right]\right|_{\mathbf{x}=\mathbf{x}^{\prime}} .
$$


The quantities (27) are expressed in terms of $S^{c}\left(x, x^{\prime}\right)$, which is the causal Green function (propagator) of the Dirac equation with an external field, and in terms of $S^{p, \bar{p}, \theta}$, which are solutions of the same equation. The quantities $\left\langle j_{\mu}(t)>^{0},<T_{\mu \nu}(t)>^{0},<j_{\mu}(t)>_{\text {out }}^{0}\right.$, and $\left\langle T_{\mu \nu}(t)>_{\text {out }}^{0}\right.$ are contributions to the corresponding mean values at zero temperature and density. The quantities $\left\langle j_{\mu}(t)\right\rangle^{\theta}$ and $<T_{\mu \nu}(t)>^{\theta}$ present the contributions due to the existence of the initial thermal distribution.

The quantities $\left\langle j_{\mu}(t)\right\rangle^{0},\left\langle T_{\mu \nu}(t)\right\rangle^{0},\left\langle j_{\mu}(t)\right\rangle_{\text {out }}^{0},\left\langle T_{\mu \nu}(t)\right\rangle_{\text {out }}^{0},\left\langle j_{\mu}(t)\right\rangle^{\theta}$, and $\left\langle T_{\mu \nu}(t)\right\rangle^{\theta}$ are real-valued by construction, due to the properties of the singular functions $S_{i n}^{c}, S_{\text {out }}^{c}$ and $S^{\theta}$. On the contrary, the quantities $\left\langle j_{\mu}(t)\right\rangle^{c, p, \bar{p}}$ and $\left\langle T_{\mu \nu}(t)\right\rangle^{c, p, \bar{p}}$ are not necessarily real-valued. For the purpose of the following consideration, it is useful to rewrite $\left\langle j_{\mu}(t)\right\rangle^{0}$ and $\left\langle T_{\mu \nu}(t)\right\rangle^{0}$ in the form

$$
\begin{aligned}
\left\langle j_{\mu}(t)\right\rangle^{0}= & \operatorname{Re}\left\langle j_{\mu}(t)\right\rangle^{c}+\operatorname{Re}\left\langle j_{\mu}(t)\right\rangle^{p}, \\
\left\langle T_{\mu \nu}(t)\right\rangle^{0}= & \operatorname{Re}\left\langle T_{\mu \nu}(t)\right\rangle^{c}+\operatorname{Re}\left\langle T_{\mu \nu}(t)\right\rangle^{p}, \\
\left\langle j_{\mu}(t)\right\rangle_{\text {out }}^{0}= & \operatorname{Re}\left\langle j_{\mu}(t)\right\rangle^{c}+\operatorname{Re}\left\langle j_{\mu}(t)\right\rangle^{\bar{p}} \\
\left\langle T_{\mu \nu}(t)\right\rangle_{\text {out }}^{0}= & \operatorname{Re}\left\langle T_{\mu \nu}(t)\right\rangle^{c}+\operatorname{Re}\left\langle T_{\mu \nu}(t)\right\rangle^{\bar{p}} .
\end{aligned}
$$

\section{The initial state as vacuum}

\subsection{T-constant field regularization}

In this section, we examine the quantities $\left\langle j_{\mu}(t)>^{0}\right.$ and $<T_{\mu \nu}(t)>^{0}$ that represent contributions to the corresponding mean values at zero temperature. In order to calculate such quantities, the $T$-constant field regularization is necessary. In particular, we are going to study the leading contributions to the divergent parts of the quantities at $T \rightarrow \infty$. To make the consideration complete, and to provide the reader with some formulas necessary for the further consideration, we start by reproducing some of the results on the mean numbers of created particle that were obtained in [28].

We chose the $T$-constant field potentials $A_{\mu}^{E}$ as follows, $A_{0}^{E}=A_{1}^{E}=A_{2}^{E}=0$ :

$$
A_{3}^{E}(t)= \begin{cases}E t_{1}, & t \in\left(-\infty, t_{1}\right), \\ E t, & t \in\left[t_{1}, t_{2}\right] \\ E t_{2}, & t \in\left(t_{2},+\infty\right),\end{cases}
$$

where $t_{2}=-t_{1}=T / 2$. The corresponding electric field $E(t)$ is given by

$$
E(t)= \begin{cases}0, & t \in\left(-\infty, t_{1}\right), \\ E, & t \in\left[t_{1}, t_{2}\right] \\ 0, & t \in\left(t_{2},+\infty\right),\end{cases}
$$

First, let us suppose that the magnetic field is absent.

For the purpose of $T$-regularization, it is sufficient to choose, for $\left\{{ }_{\zeta} \psi_{n}(x)\right\}$ and $\left\{{ }^{\zeta} \psi_{n}(x)\right\}$, defined above, the following orthonormalized sets of solutions of the Dirac equation with a constant electric field:

$$
\begin{aligned}
& { }_{ \pm} \psi_{\mathbf{p}, r}(x)=(\gamma P+M)_{ \pm} \phi_{\mathbf{p}, \pm 1, r}(x),{ }^{ \pm} \psi_{\mathbf{p}, r}(x)=(\gamma P+M)^{ \pm} \phi_{\mathbf{p}, \mp 1, r}(x), \\
& { }_{ \pm} \phi_{\mathbf{p}, s, r}(x)={ }_{ \pm} \phi_{\mathbf{p}, s}(t) \exp \{i \mathbf{p x}\} v_{s, r}, \quad{ }^{ \pm} \phi_{\mathbf{p}, s, r}(x)={ }^{ \pm} \phi_{\mathbf{p}, s}(t) \exp \{i \mathbf{p x}\} v_{s, r}, \\
& { }_{+} \phi_{\mathbf{p}, s}(t)=C D_{\nu-\frac{1+s}{2}}( \pm(1-i) \xi),{ }_{-}^{+} \phi_{\mathbf{p}, s}(t)=C D_{-\nu-\frac{1-s}{2}}( \pm(1+i) \xi), s= \pm 1,
\end{aligned}
$$

where $\mathbf{p}$ is the momentum and $r= \pm 1$ is the spin projection; $D_{\nu}(z)$ is the Weber parabolic cylinder (WPC) function [34, and

$$
\begin{aligned}
& \nu=\frac{i \lambda}{2}, \lambda=\frac{M^{2}+\mathbf{p}_{\perp}^{2}}{|q E|}, \mathbf{p}_{\perp}=\left(p^{1}, p^{2}, 0\right), \quad \xi=\xi(t)=\frac{q E t-p_{3}}{\sqrt{|q E|}} \operatorname{sgn}(q E), \\
& C=(2 \pi)^{-3 / 2}(2|q E|)^{-1 / 2} \exp (-\pi \lambda / 8),
\end{aligned}
$$

$v_{s, r}$ are constant orthonormal spinors, $v_{s, r}^{\dagger} v_{s, r^{\prime}}=\delta_{r, r^{\prime}}$, subject to the supplementary condition $(1 \pm$ $\left.\gamma^{0} \gamma^{3}\right) v_{\mp 1, r}=0$. Using an asymptotic expansion of the WPC-function [34,

$$
D_{\nu}(z)=z^{\nu} \exp \left(-\frac{z^{2}}{4}\right)\left(\sum_{n=0}^{N} \frac{\left(-\frac{1}{2} \nu\right)_{n}\left(\frac{1}{2}-\frac{1}{2} \nu\right)_{n}}{n !\left(-\frac{1}{2} z^{2}\right)^{n}}+O\left(|z|^{-2(N+1)}\right)\right),|\arg z|<3 \pi / 4,
$$

we can obtain the asymptotics of particle energies as $T \rightarrow \infty$,

$$
\varepsilon_{\mathbf{p}}^{(\zeta)}=\left|\frac{q E T}{2}+p_{3}\right|, \quad \tilde{\varepsilon}_{\mathbf{p}}^{(\zeta)}=\left|\frac{q E T}{2}-p_{3}\right| .
$$


One can see that the matrices $G\left(\zeta^{\zeta^{\prime}}\right)$ (7) are diagonal:

$$
G\left(\left.{ }_{\zeta}\right|^{\zeta^{\prime}}\right)_{\mathbf{p}, r, \mathbf{p}^{\prime}, r^{\prime}}=\delta_{r, r^{\prime}} \delta\left(\mathbf{p}-\mathbf{p}^{\prime}\right) g\left(\left.{ }_{\zeta}\right|^{\zeta^{\prime}}\right) .
$$

The differential mean numbers of electrons (equal to the corresponding differential mean number of pairs) with a given momentum $\mathbf{p}$ and spin projections $r$ created from vacuum are

$$
\left.\aleph_{\mathbf{p}, r}=\langle 0, \text { in }| a_{\mathbf{p}, r}^{\dagger}(\text { out }) a_{\mathbf{p}, r}(\text { out }) \mid 0, \text { in }\right\rangle=\left|g\left(-\left.\right|^{+}\right)\right|^{2},
$$

were the standard volume regularization is used, so that $\delta\left(\mathbf{p}-\mathbf{p}^{\prime}\right) \rightarrow \delta_{\mathbf{p}, \mathbf{p}^{\prime}}$. Note that $\aleph_{\mathbf{p}, r}$ is even with respect to the sign of $q E$.

If the time $T$ is sufficiently large, $T>>T_{0}=(1+\lambda) / \sqrt{|q E|}$, the differential mean numbers $\aleph_{\mathbf{p}, r}$ have the form

$$
\aleph_{\mathbf{p}, r}=\left\{\begin{array}{l}
e^{-\pi \lambda}\left[1+O\left(\left[\frac{1+\lambda}{K_{p}}\right]^{3}\right)\right],-\sqrt{|q E|} \frac{T}{2} \leq \bar{\xi} \leq-K_{p}, \\
O(1),-K_{p}<\bar{\xi} \leq K_{p}, \\
O\left(\left[\frac{1+\lambda}{\xi^{2}}\right]^{3}\right), \bar{\xi}>K_{p},
\end{array}\right.
$$

where

$$
\bar{\xi}=\frac{\left|p_{3}\right|-|q E| T / 2}{\sqrt{|q E|}},
$$

and $K_{p}$ is a sufficiently large arbitrary constant, $K_{p}>>1+\lambda$; see [28]. In the limit $T \rightarrow \infty$, the differential mean numbers have a simple form:

$$
\aleph_{\mathbf{p}, r}=e^{-\pi \lambda},
$$

which is identical with the one obtained for a constant electric field by Nikishov 33 . One can see that the stabilization of the differential mean numbers to the asymptotic form (36) for finite longitudinal momenta is reached at $T>>T_{0}$. The characteristic time $T_{0}$ is called the stabilization time.

In order to study the effects of switching the electric field on and off, at $T>>T_{0}$, one can study another example of a quasiconstant electric field:

$$
E(t)=E \cosh ^{-2}\left(\frac{t}{\alpha}\right) .
$$

This field switches on and off adiabatically as $t \rightarrow \pm \infty$ and is quasi-constant at finite times. The differential mean numbers of particles created by such a field have been found in [35. As shown in 28, the differential mean numbers in the field (37) have the asymptotic form (36) for sufficiently large $\alpha$, $\alpha>\alpha_{0}=(1+\sqrt{\lambda}) / \sqrt{|q E|}$ and for $|q E| \alpha \gg\left|p_{3}\right|$. Thus, $\alpha_{0}$ can be interpreted as the stabilization time for such a field. At the same time, the latter implies that the effects of switching on and off are not essential at large times and finite longitudinal momenta for both fields. Extrapolating this conclusion, one may suppose that in any electric field being quasiconstant $\approx E$ at least for a time period $T>T_{0}$ and switching on and off outside this period particle-creation effects have no dependence on the details of switching on and off. Therefore, calculations in a $T$-constant field are representative for a large class of quasiconstant electric fields.

\subsection{Contributions due to particle-creation}

Let us apply the above results to a calculation of the leading terms in $\operatorname{Re}\left\langle j_{\mu}(t)\right\rangle^{p, \bar{p}}$ and $\operatorname{Re}\left\langle T_{\mu \nu}(t)\right\rangle^{p, \bar{p}}$ from (28) for sufficiently large times $t$, more exactly, for large values of $t-t_{1}=t+T / 2$, and of $T$. Being written in a dimensionless form these two conditions have the form

$$
\begin{aligned}
& \sqrt{|q E|}(t+T / 2) \gg 1+M^{2} /|q E|, \quad t \leq T / 2, \\
& \sqrt{|q E| T} \gg 1+M^{2} /|q E|, \quad t>T / 2 .
\end{aligned}
$$

We note that the dimensionless parameter

$$
\sqrt{|q E|} T=\sqrt{\frac{c}{\hbar}|q E| T}
$$

that has emerged here will play an important role in the further considerations, and will enter all the consistency conditions that are obtained in this article. 
We can see that (38) is a stabilization condition when the leading terms do not depend on the details of switching on and off. As follows from (27), we need the singular functions $S^{p}(23)$ and $S^{\bar{p}}(25)$ at $x \approx x^{\prime}$ in such an approximation, which provides the required contribution for $\operatorname{Re}\left\langle j_{\mu}(t)\right\rangle^{p, \bar{p}}$ and $\operatorname{Re}\left\langle T_{\mu \nu}(t)\right\rangle^{p, \bar{p}}$ when $(38)$ is valid. Time-dependence arises due to integration over $p_{3}$ under the condition $-\sqrt{|q E|} \frac{T}{2} \leq \bar{\xi} \leq-K$ with the subsidiary condition that $|\xi(t)|$ must be sufficiently large, $|\xi(t)| \geq K$, where $K$ is subject to the condition $K \gg 1+m^{2} /|q E|$. The distribution $\aleph_{\mathbf{p}, r}$ plays the role of a cut-off factor in the integral over $p_{\perp}$, the $T$-dependent contribution of $S^{p, \bar{p}}$ being thus convergent.

as

The range of integration over momenta in $S^{p}$ that determines the leading contributions can be defined

$$
D:\left\{\begin{array}{c}
\left|\mathbf{p}_{\perp}\right| \leq \sqrt{|q E|}[\sqrt{|q E|}(t+T / 2)-K]^{1 / 2} \\
-T / 2+K / \sqrt{|q E|} \leq p_{3} / q E \leq t-K / \sqrt{|q E|}
\end{array} .\right.
$$

Using (77) to express the solutions ${ }_{ \pm} \psi_{n}$ via ${ }^{ \pm} \psi_{n}$, and taking into account the asymptotic expansion (31), we can calculate the leading contributions to the function $S^{p}\left(x, x^{\prime}\right)$ (23),

$$
S^{p}\left(x, x^{\prime}\right)=-i \int_{D} d \mathbf{p} \sum_{r= \pm 1} \aleph_{\mathbf{p}, r}\left[{ }^{+} \psi_{\mathbf{p}, r}(x)^{+} \bar{\psi}_{\mathbf{p}, r}\left(x^{\prime}\right)-{ }^{-} \psi_{\mathbf{p}, r}(x)^{-} \bar{\psi}_{\mathbf{p}, r}\left(x^{\prime}\right)\right] .
$$

Taking summation over $r$, we represent (39) as follows:

$$
\begin{aligned}
S^{p}\left(x, x^{\prime}\right) & =(\gamma P+M) \Delta^{p}\left(x, x^{\prime}\right) \\
\Delta^{p}\left(x, x^{\prime}\right) & =-i \int_{D} \aleph_{\mathbf{p}, 0} \exp \left\{i \mathbf{p}\left(\mathbf{x}-\mathbf{x}^{\prime}\right)\right\}\left[{ }^{+} \phi_{\mathbf{p}, 0}(t)^{+} \phi_{\mathbf{p}, 0}^{*}\left(t^{\prime}\right)+{ }^{-} \phi_{\mathbf{p}, 0}(t)^{-} \phi_{\mathbf{p}, 0}^{*}\left(t^{\prime}\right)\right] d \mathbf{p} .
\end{aligned}
$$

Then, integrating over $\mathbf{p}_{\perp}$ in (40), we obtain

$$
\Delta^{p}\left(x, x^{\prime}\right)=-i \int_{-T / 2+K / \sqrt{|q E|}}^{t-K / \sqrt{|q E|}} h_{\|}\left(x_{\|}, x_{\|}^{\prime}\right) h_{\perp}\left(\mathbf{x}_{\perp}, \mathbf{x}_{\perp}^{\prime}\right) d\left(\frac{p_{3}}{q E}\right)
$$

where

$$
\begin{aligned}
& h_{\|}\left(x_{\|}, x_{\|}^{\prime}\right)=\frac{|q E|}{2 \pi\left|q E t-p_{3}\right|} \exp \left[i p_{3}\left(x_{3}-x_{3}^{\prime}\right)\right] \cos \left\{\frac{1}{2}\left[\xi\left(t^{\prime}\right)^{2}-\xi(t)^{2}\right]\right\}, \\
& h\left(\mathbf{x}_{\perp}, \mathbf{x}_{\perp}^{\prime}\right)=(2 \pi)^{-2}|q E| \exp \left(-\frac{\pi m^{2}}{|q E|}-\frac{\left(\mathbf{x}_{\perp}-\mathbf{x}_{\perp}^{\prime}\right)^{2}|q E|}{4 \pi}\right),
\end{aligned}
$$

and the notation $x_{\|}^{\mu}=\left(x^{0}, 0,0, x^{3}\right)$ and $\mathbf{x}_{\perp}=\left(x^{1}, x^{2}, 0\right)$ has been used.

In the same manner, we calculate the leading contributions to the function $S^{\bar{p}}\left(x, x^{\prime}\right)$ (25),

$$
\begin{aligned}
S^{\bar{p}}\left(x, x^{\prime}\right) & =(\gamma P+M) \Delta^{\bar{p}}\left(x, x^{\prime}\right) \\
\Delta^{\bar{p}}\left(x, x^{\prime}\right) & \left.=-i \int_{\bar{D}} \aleph_{\mathbf{p}, 0} \exp \left\{i \mathbf{p}\left(\mathbf{x}-\mathbf{x}^{\prime}\right)\right\}{ }_{+} \phi_{\mathbf{p}, 0}(t)_{+} \phi_{\mathbf{p}, 0}^{*}\left(t^{\prime}\right)+_{-} \phi_{\mathbf{p}, 0}(t)_{-} \phi_{\mathbf{p}, 0}^{*}\left(t^{\prime}\right)\right] d \mathbf{p} .
\end{aligned}
$$

The range of integration $\bar{D}$ reads

$$
\bar{D}:\left\{\begin{array}{c}
\left|\mathbf{p}_{\perp}\right| \leq \sqrt{|q E|}[\sqrt{|q E|}(t+T / 2)-K]^{1 / 2} \\
t+K / \sqrt{|q E|} \leq p_{3} / q E \leq T / 2-K / \sqrt{|q E|}
\end{array} .\right.
$$

Integrating over $\mathbf{p}_{\perp}$ in (43), we find

$$
\Delta^{\bar{p}}\left(x, x^{\prime}\right)=-i \int_{t+K / \sqrt{|q E|}}^{T / 2-K / \sqrt{|q E|}} h_{\|}\left(x_{\|}, x_{\|}^{\prime}\right) h_{\perp}\left(\mathbf{x}_{\perp}, \mathbf{x}_{\perp}^{\prime}\right) d\left(\frac{p_{3}}{q E}\right),
$$

The following results will be outlined in the presence of a constant magnetic field, $B \neq 0$. For such a field, we select the nonzero potential as $A_{1}^{B}=B x^{2}$. Now, the complete set of quantum numbers that describes particles is $\left(p_{1}, n_{B}, p_{3}, r\right), n_{B}=0,1, \ldots, \lambda=\left(M^{2}+|q B|\left(2 n_{B}+1-r\right)\right)|q E|^{-1}$. The space-time 
dependent part of the function $\phi_{\mathbf{p}, s, r}(x)$, see (30), is modified as follows:

$$
\begin{aligned}
& (2 \pi)^{-3 / 2} \exp \{i \mathbf{p x}\} \rightarrow(2 \pi)^{-1 / 2} \exp \left\{-i p_{3} x^{3}\right\} \phi_{p_{1}, n_{B}, r}\left(\mathbf{x}_{\perp}\right), \\
& \phi_{p_{1}, n_{B}, r}\left(\mathbf{x}_{\perp}\right)=\left(\frac{\sqrt{|q B|}}{2^{n_{B}+1} \pi^{3 / 2} n_{B} !}\right)^{1 / 2} \exp \left\{-i p_{1} x^{1}-\frac{|q B|}{2}\left(x^{2}-\frac{p_{1}}{q B}\right)^{2}\right\} \\
& \times \mathcal{H}_{n_{B}}\left[\sqrt{|q B|}\left(x^{2}-\frac{p_{1}}{q B}\right)\right] .
\end{aligned}
$$

Here, $\mathcal{H}_{n_{B}}(x)$ are the Hermite polynomials. Then, integration over $\mathbf{p}_{\perp}$ in the above formulas must be replaced by integration over $p_{1}$ from $-\infty$ to $+\infty$ with summation over the integer quantum numbers $n_{B}$ in the interval

$$
n_{B} \leq[\sqrt{|q E|}(t+T / 2)-K]|E / 2 B| .
$$

After this, one can see that formula (41) needs only one modification. Namely, the new (in the presence of magnetic field) value of $h_{\perp}\left(\mathbf{x}_{\perp}, \mathbf{x}_{\perp}^{\prime}\right)$ reads

$$
h_{\perp}\left(\mathbf{x}_{\perp}, \mathbf{x}_{\perp}^{\prime}\right)=\frac{q B \exp \left(\pi \Sigma^{3} B / E\right)}{4 \pi \sinh (\pi B / E)} \exp \left\{-\frac{\pi M^{2}}{|q E|}-\left(\mathbf{x}_{\perp}-\mathbf{x}_{\perp}^{\prime}\right)^{2} \frac{q B}{4} \operatorname{coth}(\pi B / E)\right\} .
$$

Using (41) and (44), one can represent $\left\langle j_{\mu}(t)\right\rangle^{p, \bar{p}}$ and $\left\langle T_{\mu \nu}(t)\right\rangle^{p, \bar{p}}$ in (27) as follows:

$$
\begin{aligned}
\left\langle j_{\mu}(t)\right\rangle^{p, \bar{p}} & =\left.i q \operatorname{tr}\left[\gamma_{\mu} \gamma P \Delta^{p, \bar{p}}\left(x, x^{\prime}\right)\right]\right|_{x=x^{\prime}}, \\
\left\langle T_{\mu \nu}(t)\right\rangle^{p, \bar{p}} & =\left.i \operatorname{tr}\left[A_{\mu \nu} \gamma P \Delta^{p, \bar{p}}\left(x, x^{\prime}\right)\right]\right|_{x=x^{\prime}} .
\end{aligned}
$$

Then, using (41), (42), (44), and (46), we find

$$
\begin{aligned}
\left\langle j_{\mu}(t)\right\rangle^{p, \bar{p}} & =\left.i q P_{\mu} \operatorname{tr} \Delta^{p, \bar{p}}\left(x, x^{\prime}\right)\right|_{x=x^{\prime}}, \\
\left\langle T_{\mu \mu}(t)\right\rangle^{p, \bar{p}} & =\left.i P_{\mu}^{2} \operatorname{tr} \Delta^{p, \bar{p}}\left(x, x^{\prime}\right)\right|_{x=x^{\prime}} .
\end{aligned}
$$

The off-diagonal matrix elements of $\left\langle T_{\mu \nu}\right\rangle^{p}$ and $\left\langle T_{\mu \nu}\right\rangle^{\bar{p}}$ are all equal to zero.

Taking derivatives, calculating traces, and integrating over $p_{3}$, we obtain the leading contributions at large $T$. First of all,

$$
\begin{aligned}
& \left\langle j_{\mu}(t)\right\rangle^{p}=-2 \delta_{\mu}^{3} q \operatorname{sgn}(q E)(1 / 2+t / T) n^{c r}, \\
& \left\langle j_{\mu}(t)\right\rangle^{\bar{p}}=-2 \delta_{\mu}^{3} q \operatorname{sgn}(q E)(1 / 2-t / T) n^{c r}, \\
& \left\langle T_{00}(t)\right\rangle^{p}=\left\langle T_{33}(t)\right\rangle^{p}=|q E| T(1 / 2+t / T)^{2} n^{c r}, \\
& \left\langle T_{00}(t)\right\rangle^{\bar{p}}=\left\langle T_{33}(t)\right\rangle^{\bar{p}}=|q E| T(1 / 2-t / T)^{2} n^{c r},
\end{aligned}
$$

where

$$
n^{c r}=\frac{q^{2}}{4 \pi^{2}} E B T \operatorname{coth}(\pi B / E)\left[\exp \left(-\pi \frac{M^{2}}{|q E|}\right)+O\left(\frac{K}{\sqrt{|q E| T}}\right)\right] .
$$

Secondly,

$$
\begin{aligned}
& \left\langle T_{11}(t)\right\rangle^{p}=\left\langle T_{22}(t)\right\rangle^{p},\left\langle T_{11}(t)\right\rangle^{\bar{p}}=\left\langle T_{22}(t)\right\rangle^{\bar{p}}, \\
& \left\langle T_{11}(t)\right\rangle^{p}=\widetilde{n}\left\{\begin{array}{l}
\ln [\sqrt{|q E|}(T / 2+t)]+O(\ln K), \sqrt{|q E|}(T / 2+t)>K \\
O(\ln K), \sqrt{|q E|}(T / 2+t) \leq K
\end{array}\right. \\
& \left\langle T_{11}(t)\right\rangle^{\bar{p}}=\widetilde{n}\left\{\begin{array}{l}
-\ln [\sqrt{|q E|}(T / 2-t)]+O(\ln K), \sqrt{|q E|}(T / 2-t)>K \\
-O(\ln K), \sqrt{|q E|}(T / 2-t) \leq K
\end{array}\right.
\end{aligned}
$$

where

$$
\widetilde{n}=\frac{(q B)^{2}}{4 \pi^{2} \sinh ^{2}(\pi B / E)} \exp \left(-\pi \frac{M^{2}}{|q E|}\right) .
$$

We can see that all the leading contributions are real-valued. Note that the current density $\left\langle j_{\mu}(t)\right\rangle^{p}$ and the component of EMT $\left\langle T_{\mu \nu}(t)\right\rangle^{p}$ are zero for $t \leq t_{1}$ and change with time until $t_{2},\left\langle j_{3}(t)\right\rangle^{p}$ being linear, $\left\langle T_{00}(t)\right\rangle^{p}=\left\langle T_{33}(t)\right\rangle^{p}$ quadratic, and $\left\langle T_{11}(t)\right\rangle^{p}=\left\langle T_{22}(t)\right\rangle^{p}$ logarithmic, respectively. As will be 
demonstrated, the rate $n^{c r}$ in (48) is the total number-density of pairs created by the $T$-constant electric field.

After switching on of the electric field (at $t>t_{2}$ ), all the mean values (48), (50) are constant and retain their values at $t_{2}$.

We should mention that formulas (48), (50) have been obtained for the first time.

At large times $t-t_{1}=t+T / 2 \gg K / \sqrt{|q E|}$, it is useful to examine the quantities

$$
j_{\mu}^{c r}(t)=\left\langle j_{\mu}(t)\right\rangle^{0}-\left\langle j_{\mu}(t)\right\rangle_{\text {out }}^{0}, T_{\mu \nu}^{c r}(t)=\left\langle T_{\mu \nu}(t)\right\rangle^{0}-\left\langle T_{\mu \nu}(t)\right\rangle_{\text {out }}^{0},
$$

which can be interpreted as those corresponding to out-particles at any large $t$. At the final time instant $t_{2}$, they coincide with the physical quantities $\left\langle j_{\mu}\left(t_{2}\right)\right\rangle^{p}$ and $\left\langle T_{\mu \nu}\left(t_{2}\right)\right\rangle^{p}$. Then it follows from (26) and (48), (50) that

$$
\begin{aligned}
& j_{\mu}^{c r}(t)=\left\langle j_{\mu}(t)\right\rangle^{p}-\left\langle j_{\mu}(t)\right\rangle^{\bar{p}}=-2 \delta_{\mu}^{3} q \operatorname{sgn}(q E)(2 t / T) n^{c r} \\
& T_{\mu \nu}^{c r}(t)=\left\langle T_{\mu \nu}(t)\right\rangle^{p}-\left\langle T_{\mu \nu}(t)\right\rangle^{\bar{p}}
\end{aligned}
$$

and

$$
\begin{aligned}
\left\langle T_{00}(t)\right\rangle^{c r} & =\left\langle T_{33}(t)\right\rangle^{c r}=2|q E| t n^{c r},\left\langle T_{11}(t)\right\rangle^{c r}=\left\langle T_{22}(t)\right\rangle^{c r}, \\
\left\langle T_{11}(t)\right\rangle^{c r} & =\widetilde{n}\left\{\begin{array}{l}
\ln \left[|q E|\left((T / 2)^{2}-t^{2}\right)\right]+O(\ln K), \sqrt{|q E|}(T / 2-t)>K \\
\ln [\sqrt{|q E|}(T / 2+t)]+O(\ln K), \sqrt{|q E|}(T / 2-t) \leq K
\end{array} .\right.
\end{aligned}
$$

At $t \geq t_{2}$, the production of pairs terminates, and the quantities (53) and (54) maintain their values (53) and (54) at $t=t_{2}$, and present the current density and EMT of created particles. For example, from $j_{\mu}^{c r}$ in (53) we can see that $n^{c r}$ is the total number-density of pairs created during the entire time of action of the electric field.

We emphasize that expressions (48), (150) for $\left\langle j_{\mu}(t)\right\rangle^{p}$ and $\left\langle T_{\mu \nu}(t)\right\rangle^{p}$ represent contributions to $\left\langle j_{\mu}(t)\right\rangle^{0}$ and $\left\langle T_{\mu \nu}(t)\right\rangle^{0}$, respectively, at any time instant $t$ if the stabilization condition (38) is valid. On the contrary, expressions (53), (54) are valid only for time instants $\bar{t}$ that are sufficiently close to $t_{2}$,

$$
\bar{t}:(T-2 \bar{t}) / T \ll 1,
$$

when the interpretation in terms of final particles already makes sense.

\subsection{Vacuum polarization contributions}

We are now going to calculate the real-valued parts of the quantities $\left\langle j_{\mu}(t)\right\rangle^{c}$ and $\left\langle T_{\mu \nu}(t)\right\rangle^{c}$ defined in (27), which do not have any $T$-divergences. In these expressions, we can use the causal Green function $S^{c}\left(x, x^{\prime}\right)(22)$ in the constant field, since the above quantities do not need any regularization.

For such a Green function, the so-called Fock-Schwinger proper time representation holds true:

$$
S^{c}\left(x, x^{\prime}\right)=(\gamma P+M) \Delta^{c}\left(x, x^{\prime}\right), \Delta^{c}\left(x, x^{\prime}\right)=\int_{0}^{\infty} f\left(x, x^{\prime}, s\right) d s ;
$$

see [33] and [36], where the Fock-Schwinger kernel $f\left(x, x^{\prime}, s\right)$ reads

$$
\begin{aligned}
f\left(x, x^{\prime}, s\right) & =\exp \left(-i \frac{q}{2} \sigma^{\mu \nu} F_{\mu \nu} s\right) f^{(0)}\left(x, x^{\prime}, s\right), \sigma^{\mu \nu}=\frac{i}{2}\left[\gamma^{\mu}, \gamma^{\nu}\right] \\
f^{(0)}\left(x, x^{\prime}, s\right) & =\frac{q^{2} E B \exp (i q \Lambda)}{(4 \pi)^{2} \sinh (q E s) \sin (q B s)} \exp \left[-i M^{2} s-i \frac{\left(x-x^{\prime}\right) q F \operatorname{coth}(q F s)\left(x-x^{\prime}\right)}{4}\right]
\end{aligned}
$$

see [8, 37. Singularities of the kernel are situated at the origin and on the imaginary axis, $|q E| s=-i \pi n$, $n=1,2,3, \ldots$.

It should be noted that in this expression the only term

$$
\Lambda=\Lambda_{\|}+\Lambda_{\perp}, \quad \Lambda_{\|}=\left(x_{0}+x_{0}^{\prime}\right)\left(x_{3}-x_{3}^{\prime}\right) E / 2, \quad \Lambda_{\perp}=-\int_{x^{\prime}}^{x} A_{\mu}^{B} d x^{\mu},
$$

is potential-dependent. Here, $A_{\mu}^{B}$ is the potential of magnetic field $F_{\mu \nu}^{B}$, and the integral is taken along the line connecting the points $x$ and $x^{\prime}$.

Using this representation, we calculate $\operatorname{Re}\left\langle j_{\mu}(t)\right\rangle^{c}$ and $\operatorname{Re}\left\langle T_{\mu \nu}(t)\right\rangle^{c}$. It is easy to see that $\left\langle j_{\mu}(t)\right\rangle^{c}=0$, as should be expected due to translational symmetry, and $\left\langle T_{\mu \nu}(t)\right\rangle^{c}=0, \mu \neq \nu$. 
Calculating the diagonal terms $\left\langle T_{\mu \mu}(t)\right\rangle^{c}$, we discover that it can be derived from the Heisenberg-Euler Lagrangian $\mathcal{L}$,

$$
\mathcal{L}=\frac{1}{2} \operatorname{tr} \int_{0}^{\infty} s^{-1} f(x, x, s) d s .
$$

Subtracting the zero external field contribution from $\mathcal{L}$ and performing the standard renormalizations of $\mathcal{L}-\left.\mathcal{L}\right|_{F=0}$, that leave $q F_{\mu \nu}$ invariant, i.e., the standard renormalizations of the coupling constant $q^{2}$ and potentials $A_{\mu}$, we obtain a finite expression:

$$
\mathcal{L}_{\text {ren }}=\int_{0}^{\infty} \frac{d s \exp \left(-i M^{2} s\right)}{8 \pi^{2} s}\left[q^{2} E B \operatorname{coth}(q E s) \cot (q B s)-\frac{1}{s^{2}}-\frac{q^{2}}{3}\left(E^{2}-B^{2}\right)\right] ;
$$

see [7. Making the same renormalization for $\left\langle T_{\mu \mu}(t)\right\rangle^{c}$, we can see that there holds the relation

$$
\begin{aligned}
& \left\langle T_{00}(t)\right\rangle_{\text {ren }}^{c}=-\left\langle T_{33}(t)\right\rangle_{\text {ren }}^{c}=E \frac{\partial \mathcal{L}(t)_{\text {ren }}}{\partial E}-\mathcal{L}(t)_{r e n}, \\
& \left\langle T_{11}(t)\right\rangle_{\text {ren }}^{c}=\left\langle T_{22}(t)\right\rangle_{\text {ren }}^{c}=\mathcal{L}(t)_{r e n}-B \frac{\partial \mathcal{L}(t)_{\text {ren }}}{\partial B},
\end{aligned}
$$

where $\mathcal{L}(t)_{r e n}=\left.\mathcal{L}_{r e n}\right|_{E \rightarrow E(t)}$, and $E(t)$ is defined by (29).

Finally, the vacuum mean values of the current density and EMT have the form

$$
\left\langle j_{\mu}(t)\right\rangle^{0}=\operatorname{Re}\left\langle j_{\mu}(t)\right\rangle^{p},\left\langle T_{\mu \nu}(t)\right\rangle_{r e n}^{0}=\operatorname{Re}\left\langle T_{\mu \nu}(t)\right\rangle_{\text {ren }}^{c}+\operatorname{Re}\left\langle T_{\mu \nu}(t)\right\rangle^{p},
$$

where $\operatorname{Re}\left\langle j_{\mu}(t)\right\rangle^{p}$ and $\operatorname{Re}\left\langle T_{\mu \nu}(t)\right\rangle^{p}$ are presented by (48) and (50). We can see that the $T$-dependent contributions $\operatorname{Re}\left\langle j_{\mu}(t)\right\rangle^{p}$ and $\operatorname{Re}\left\langle T_{\mu \nu}(t)\right\rangle^{p}$ arise due to vacuum instability; they are global physical quantities and have the factor $\exp \left\{-\pi M^{2} /|q E|\right\}$. This factor is exponentially small for a weak electric field, $M^{2} /|q E| \gg 1$, and these quantities can actually be observed as soon as the external field strength approaches the critical value $E_{c}$. On the other hand, the term $\operatorname{Re}\left\langle T_{\mu \nu}(t)\right\rangle_{\text {ren }}^{c}$ is $t$-dependent and $T$ independent; it is therefore local and does exist in arbitrary electric fields. When the $T$-constant electric field switches off, the local contribution from the electric field to $\operatorname{Re}\left\langle T_{\mu \nu}(t)\right\rangle_{\text {ren }}^{c}$ vanishes, but the global one, given by $\operatorname{Re}\left\langle T_{\mu \nu}\left(t_{2}\right)\right\rangle^{p}$, does remain. Thus, in the general case, both kinds of contributions are important. We stress that in order to make $\left\langle T_{\mu \nu}(t)\right\rangle_{\text {ren }}^{0}$ finite the only term $\left\langle T_{\mu \nu}(t)\right\rangle^{c}$ has to be regularized and renormalized due to the standard ultraviolet divergences, which is consistent with the fact that the ultraviolet divergences have a local nature.

Using expression (60), we can find a condition which imposes restrictions on the strength of classical constant electric field. Note that for a strong electric field $(B=0), M^{2} /|q E| \ll 1$, and for a large $T$, there is a well-known asymptotic expression for the vacuum energy density $\operatorname{Re}\left\langle T_{00}(t)\right\rangle_{r e n}^{c}$,

$$
\operatorname{Re}\left\langle T_{00}(t)\right\rangle_{r e n}^{c}=-\frac{q^{2}}{24 \pi^{2}} E^{2} \ln \frac{|q E|}{M^{2}} .
$$

It does not depend on $T$. The energy density of a classical electric field is $E^{2} / 8 \pi$. Then (see [38]) one makes the conclusion that the notion of a strong constant electric field is physically meaningful when (61) is much less than $E^{2} / 8 \pi$, which implies

$$
\frac{q^{2}}{3 \pi} \ln \frac{|q E|}{M^{2}} \ll 1 .
$$

However, if $T$ is large, one also has to take into account the $T$-dependent term $\operatorname{Re}\left\langle T_{00}(t)\right\rangle^{p}$ in (60). At $t=t_{2}$, and for an arbitrary magnitude of the electric field, we have

$$
\operatorname{Re}\left\langle T_{00}\left(t_{2}\right)\right\rangle^{p}=\frac{q^{2} E^{2}}{4 \pi^{3}}|q E| T^{2} \exp \left(-\pi \frac{M^{2}}{|q E|}\right) .
$$

\footnotetext{
${ }^{4}$ In the absence of electric field, the Green function $S^{c}\left(x, x^{\prime}\right), S_{i n}^{c}\left(x, x^{\prime}\right)$, and $S_{\text {out }}^{c}\left(x, x^{\prime}\right)$ coincide. Therefore, in case $t<t_{1}$ we have $\left\langle T_{\mu \nu}(t)\right\rangle^{0}=.\left.\left\langle T_{\mu \nu}(t)\right\rangle^{0}\right|_{E=0}=\left.\left\langle T_{\mu \nu}(t)\right\rangle^{c}\right|_{E=0}$. For $t>t_{2}$, relation (52) implies

$$
\left\langle T_{\mu \nu}(t)\right\rangle^{0}=\left\langle T_{\mu \nu}(t)\right\rangle_{o u t}^{0}+T_{\mu \nu}^{c r}(t)
$$

where $T_{\mu \nu}^{c r}(t)$ is given by (53), (54) and $\left\langle T_{\mu \nu}(t)\right\rangle_{o u t}^{0}=.\left.\left\langle T_{\mu \nu}(t)\right\rangle^{0}\right|_{E=0}=\left.\left\langle T_{\mu \nu}(t)\right\rangle^{c}\right|_{E=0}$, i.e., this is a vacuum polarization contribution.

${ }^{5}$ The same inequality has been obtained by Ritus as a condition to avoid the breakdown of the loop expansion in a strong field; see, e.g., [39].
} 
One can neglect the back-reaction of created pairs in electric field only in case (63) is far less than $E^{2} / 8 \pi$, which implies

$$
|q E| T^{2} \ll \frac{\pi^{2}}{2 q^{2}} \exp \left(\pi \frac{M^{2}}{|q E|}\right)
$$

or, in Gaussian units,

$$
\frac{c}{\hbar}|q E| T^{2} \ll \frac{\pi^{2} c \hbar}{2 q^{2}} \exp \left(\pi \frac{c^{3} M^{2}}{\hbar|q E|}\right) .
$$

The restriction (64) is completely new, it is much more restrictive than (62). It imposes restrictions both on the maximal strength of electric field and its duration due to the back-reaction of created particles.

\section{Initial state as thermal equilibrium}

We are now going to calculate the quantities $<j_{\mu}(t)>$ and $<T_{\mu \nu}(t)>$ (13) with the density operator (15), by using relations (26). In fact, the contributions $\left\langle j_{\mu}(t)>^{0}\right.$ and $\left\langle T_{\mu \nu}(t)>^{0}\right.$ to these quantities have been calculated in the previous section. Here, we study the remaining temperature-dependent contributions $\left\langle j_{\mu}(t)\right\rangle^{\theta}$ and $\left\langle T_{\mu \nu}(t)\right\rangle^{\theta}$ to these quantities.

\subsection{Contributions due to the particle creation}

Let us examine the function $S^{\theta}\left(x, x^{\prime}\right)$ in (19). The form of the functions $\tilde{S}_{\theta}^{\mp}$ in (19) for $t<t_{1}$, and $t^{\prime}<t_{1}$ is known, they are functions for zero electric field. Therefore, we have to study the case $t>t_{1}, t^{\prime}>t_{1}$. First of all, we separate the contributions from particle creation in the same manner as it has been done for the function $S_{i n}^{c}\left(x, x^{\prime}\right)$ in Sec. 2; for details, see Subsec. 1 of the Appendix.

Using (26), (60), and (136) (see the Appendix), we present the mean values $\left\langle j_{\mu}(t)\right\rangle$ and $\left\langle T_{\mu \nu}(t)\right\rangle_{r e n}$ :

$$
\begin{aligned}
& \left\langle j_{\mu}(t)\right\rangle=\operatorname{Re}\left\langle j_{\mu}(t)\right\rangle_{\theta}^{c}+J_{\mu}^{p}(t), \\
& J_{\mu}^{p}(t)=\operatorname{Re}\left\langle j_{\mu}(t)\right\rangle^{p}+\operatorname{Re}\left\langle j_{\mu}(t)\right\rangle_{\theta}^{p}, \\
& \left\langle T_{\mu \nu}(t)\right\rangle_{r e n}=\operatorname{Re}\left\langle T_{\mu \nu}(t)\right\rangle_{r e n}^{c}+\operatorname{Re}\left\langle T_{\mu \nu}(t)\right\rangle_{\theta}^{c}+\tau_{\mu \nu}^{p}(t), \\
& \tau_{\mu \nu}^{p}(t)=\operatorname{Re}\left\langle T_{\mu \nu}(t)\right\rangle^{p}+\operatorname{Re}\left\langle T_{\mu \nu}(t)\right\rangle_{\theta}^{p} .
\end{aligned}
$$

Now, $\left\langle T_{\mu \nu}(t)\right\rangle$ is replaced by the renormalized (with respect to ultraviolet divergences) quantity $\left\langle T_{\mu \nu}(t)\right\rangle_{\text {ren }}$ by inserting $\left\langle T_{\mu \nu}(t)\right\rangle_{\text {ren }}^{c}$ in the r.h.s. instead of $\left\langle T_{\mu \nu}(t)\right\rangle^{c}$. The terms $J_{\mu}^{p}(t)$ and $\tau_{\mu \nu}^{p}(t)$ with the upper script $p$ represent the contributions from particle-creation, the terms with the upperscript $c$ represent the remaining contributions. One ought to recall that the vacuum polarization contribution $\left\langle T_{\mu \nu}(t)\right\rangle_{\text {ren }}^{c}$ has been already calculated and has the form (59). In the absence of electric field (for $t<t_{1}$ and $\left.t\right\rangle t_{2}$ ), the quantity (65) does not depend on time, in particular, $J_{\mu}^{p}(t)=\tau_{\mu \nu}^{p}(t)=0$ for $t<t_{1}$. As has been already mentioned, the interpretation in terms of out-particles makes sense only for $t>\bar{t}$, where $\bar{t}$ is sufficiently close to $t_{2},(T-2 \bar{t}) / T \ll 1$. At the same time, for $t>\bar{t}$ the quantities $J_{\mu}^{p}(t)$ and $\tau_{\mu \nu}^{p}(t)$ are the current density and EMT of out-particles created by the electric field. The quantities $\operatorname{Re}\left\langle j_{\mu}(t)\right\rangle_{\theta}^{c}$ and $\operatorname{Re}\left\langle T_{\mu \nu}(t)\right\rangle_{\theta}^{c}$ describe the corresponding contributions related to the existence of real in-particles at the initial state.

The quantities $\operatorname{Re}\left\langle j_{\mu}(t)\right\rangle_{\theta}^{p}$ and $\operatorname{Re}\left\langle T_{\mu \nu}(t)\right\rangle_{\theta}^{p}$ present the contributions from particle-creation at finite temperature. They are $T$-divergent. The leading contributions to these quantities at large $T$ can be found by analogy with the case of zero temperature, which has been investigated above. We examine the leading contributions to $S_{\theta, \zeta}^{p}$, which are given by the expression

$$
S_{\theta, \zeta}^{p}\left(x, x^{\prime}\right)=i \int_{D} d \mathbf{p} \sum_{r= \pm 1} N_{p_{3}}^{(\zeta)}(i n) \aleph_{\mathbf{p}, r}\left({ }^{+} \psi_{\mathbf{p}, r}(x)^{+} \bar{\psi}_{\mathbf{p}, r}\left(x^{\prime}\right)-{ }^{-} \psi_{\mathbf{p}, r}(x)^{-} \bar{\psi}_{\mathbf{p}, r}\left(x^{\prime}\right)\right)
$$

The integral $\int_{D} d \mathbf{p}$ is defined in (39); the differential numbers $\aleph_{\mathbf{p}, r}$ are defined in (36), and

$$
N_{p_{3}}^{(\zeta)}(i n)=\left\{\exp \left[\beta\left(\varepsilon_{\mathbf{p}}^{(\zeta)}-\mu^{(\zeta)}\right)\right]+1\right\}^{-1},
$$

where $\varepsilon_{\mathbf{p}}^{(\zeta)}$ in (67) are quasi-energies at $t_{1}$; see (32).

In order to obtain a generalization of (66) to the presence of a constant magnetic field, $B \neq 0$, we have to follow the way described in Subsec. 3.2. Then, taking summation and integration, we obtain the 
leading contribution to the function $S_{\theta, \zeta}^{p}\left(x, x^{\prime}\right)$ in the case under consideration:

$$
\begin{aligned}
& S_{\theta, \zeta}^{p}\left(x, x^{\prime}\right)=(\gamma P+M) \Delta_{\theta, \zeta}^{p}\left(x, x^{\prime}\right), \\
& \Delta_{\theta, \zeta}^{p}\left(x, x^{\prime}\right)=i \int_{-T / 2+K / \sqrt{|q E|}}^{t-K / \sqrt{|q E|}} N_{p_{3}}^{(\zeta)}(i n) h_{\|}\left(x_{\|}, x_{\|}^{\prime}\right) h_{\perp}\left(\mathbf{x}_{\perp}, \mathbf{x}_{\perp}^{\prime}\right) d\left(\frac{p_{3}}{q E}\right) .
\end{aligned}
$$

The functions $h_{\|}\left(x_{\|}, x_{\|}^{\prime}\right)$ and $h_{\perp}\left(\mathbf{x}_{\perp}, \mathbf{x}_{\perp}^{\prime}\right)$ are given by expressions (42) and (46), respectively.

Therefore, we obtain the leading contributions to $\left\langle j_{\mu}(t)\right\rangle_{\theta}^{p}$ and $\left\langle T_{\mu \nu}(t)\right\rangle_{\theta}^{p}$, namely,

$$
\begin{aligned}
\left\langle j_{\mu}(t)\right\rangle_{\theta}^{p} & =\left.i q \sum_{\zeta= \pm} P_{\mu} \operatorname{tr} \Delta_{\theta, \zeta}^{p}\left(x, x^{\prime}\right)\right|_{x=x^{\prime}}, \\
\left\langle T_{\mu \nu}(t)\right\rangle_{\theta}^{p} & =0, \mu \neq \nu ;\left\langle T_{\mu \mu}(t)\right\rangle_{\theta}^{p}=\left.i \sum_{\zeta= \pm} P_{\mu}^{2} \operatorname{tr} \Delta_{\theta, \zeta}^{p}\left(x, x^{\prime}\right)\right|_{x=x^{\prime}} .
\end{aligned}
$$

In order to examine the temperature-dependent contributions to $J_{\mu}^{p}(t)$ and $\tau_{\mu \nu}^{p}(t)$ in (65), we have to investigate the quantities (69). At low temperatures, $\beta\left|\left(\varepsilon_{\mathbf{p}}^{(\zeta)}-\mu^{(\zeta)}\right)\right| \gg 1$, and assuming $\varepsilon_{\mathbf{p}}^{(\zeta)}>\left|\mu^{(\zeta)}\right|$, one can see that the contributions from $\operatorname{Re}\left\langle j_{\mu}(t)\right\rangle_{\theta}^{p}$ and $\operatorname{Re}\left\langle T_{\mu \nu}(t)\right\rangle_{\theta}^{p}$ are very small in comparison with the vacuum contributions $\operatorname{Re}\left\langle j_{\mu}(t)\right\rangle^{p}$ and $\operatorname{Re}\left\langle T_{\mu \nu}(t)\right\rangle^{p}$. Therefore, in the case under consideration the leading contributions to $J_{\mu}^{p}(t)$ and $\tau_{\mu \nu}^{p}(t)$ are given by

$$
J_{\mu}^{p}(t)=\operatorname{Re}\left\langle j_{\mu}(t)\right\rangle^{p}, \tau_{\mu \nu}^{p}(t)=\operatorname{Re}\left\langle T_{\mu \nu}(t)\right\rangle^{p} .
$$

For $\varepsilon_{\mathbf{p}}^{(\zeta)}<\left|\mu^{(\zeta)}\right|$, with a sufficiently large $\left|\mu^{(\zeta)}\right| \gg \sqrt{|q E|} K$, contributions from $\operatorname{Re}\left\langle j_{\mu}(t)\right\rangle_{\theta}^{p}$ and $\operatorname{Re}\left\langle T_{\mu \nu}(t)\right\rangle_{\theta}^{p}$ are comparable with contributions from $\operatorname{Re}\left\langle j_{\mu}(t)\right\rangle^{p}$ and $\operatorname{Re}\left\langle T_{\mu \nu}(t)\right\rangle^{p}$, respectively. For example, at $\left|\mu^{(\zeta)}\right| \gtrsim|q E|(t+T / 2)$ we have

$$
\operatorname{Re}\left\langle j_{\mu}(t)\right\rangle_{\theta}^{p}=-2 \operatorname{Re}\left\langle j_{\mu}(t)\right\rangle^{p}, \operatorname{Re}\left\langle T_{\mu \nu}(t)\right\rangle_{\theta}^{p}=-2 \operatorname{Re}\left\langle T_{\mu \nu}(t)\right\rangle^{p} .
$$

Then

$$
J_{\mu}^{p}(t)=-\operatorname{Re}\left\langle j_{\mu}(t)\right\rangle^{p}, \tau_{\mu \nu}^{p}(t)=-\operatorname{Re}\left\langle T_{\mu \nu}(t)\right\rangle^{p} .
$$

The negative sign in these expressions implies that the total number-density of particles decreases due to electron-positron annihilation in electric field, and, as a consequence, $\left\langle j_{\mu}(t)\right\rangle$ and $\left\langle T_{\mu \nu}(t)\right\rangle_{\text {ren }}$ in (65) are less than $\operatorname{Re}\left\langle j_{\mu}(t)\right\rangle_{\theta}^{c}$ and $\left\langle T_{\mu \nu}(t)\right\rangle_{\text {ren }}^{c}+\operatorname{Re}\left\langle T_{\mu \nu}(t)\right\rangle_{\theta}^{c}$, respectively. This fact is in agreement with the fact that, due to particle creation, there holds the relation

$$
N_{m}^{(\zeta)}(\text { out })=\left(1-\aleph_{m}\right) N_{m}^{(\zeta)}(\text { in })+\aleph_{m}\left[1-N_{m}^{(-\zeta)}(\text { in })\right] .
$$

between the initial $N_{m}^{(\zeta)}(i n)$ and final $N_{m}^{(\zeta)}$ (out) differential mean numbers; see 22. Thus, the differential mean numbers of created particles are given by the difference $\Delta N_{m}^{(\zeta)}=N_{m}^{(\zeta)}($ out $)-N_{m}^{(\zeta)}($ in $)$. As a result, $\Delta N_{m}^{(\zeta)}$ is negative in case $N_{m}^{(+)}($in $)+N_{m}^{(-)}($in $)>1$.

At high temperatures, $\beta\left[|q E|(t+T / 2)-\mu^{( \pm)}\right] \ll 1$, we find

$$
\begin{aligned}
& J_{\mu}^{p}(t)=\frac{\beta}{2}\left[\frac{1}{2}\left\{|q E|(t+T / 2)-\mu^{(+)}-\mu^{(-)}\right\}+\sqrt{|q E|} O(K)\right] \operatorname{Re}\left\langle j_{\mu}(t)\right\rangle^{p}, \\
& \tau_{00}^{p}(t)=\tau_{33}^{p}(t)=\frac{\beta}{2}\left[\frac{1}{3}|q E|(t+T / 2)-\frac{1}{2}\left(\mu^{(+)}+\mu^{(-)}\right)+\sqrt{|q E|} O(K)\right] \operatorname{Re}\left\langle T_{00}(t)\right\rangle^{p}, \\
& \tau_{11}^{p}(t)=\tau_{22}^{p}(t)=\frac{\beta}{2}\left[|q E|(t+T / 2)-\frac{1}{2}\left(\mu^{(+)}+\mu^{(-)}\right)\right] \\
& \times\left[1+\sqrt{|q E|} O\left(\frac{\ln K}{\ln [\sqrt{|q E|}(t+T / 2)]}\right)\right] \operatorname{Re}\left\langle T_{11}(t)\right\rangle^{p} .
\end{aligned}
$$

We can see that all the components of $J_{\mu}^{p}(t)$ and $\tau_{\mu \nu}^{p}(t)$ at high temperatures are far less than the corresponding components of the vacuum contributions $\left\langle j_{\mu}(t)\right\rangle^{p}$ and $\left\langle T_{\mu \nu}(t)\right\rangle^{p}$, which is natural for Fermi particles. 
The resulting expressions for the mean energy density of created particles make it possible to conclude that the restriction (64) for the external constant electric field, established for vacuum, is also valid at the initial low-temperature state. In case the temperature of the initial state is sufficiently high in comparison with the mean kinetic energy of created particles, $\beta|q E| T \ll 1$, this restriction changes considerably. In this case, the mean energy density of created particles after the electric field turns off,

$$
\tau_{00}^{p}\left(t_{2}\right)=\frac{1}{6} \beta|q E| T \operatorname{Re}\left\langle T_{00}\left(t_{2}\right)\right\rangle^{p},
$$

in accordance with (72), is much smaller than the corresponding vacuum expression $\operatorname{Re}\left\langle T_{00}\left(t_{2}\right)\right\rangle^{p}$ in $(63)$. Repeating the arguments of Subsec. 3.3, we find that one can neglect the back-reaction of created pairs in electric field only in case $\tau_{00}^{p}\left(t_{2}\right)$ is far less than $E^{2} / 8 \pi$, which implies

$$
\beta|q E|^{2} T^{3} \ll \frac{3 \pi^{2}}{q^{2}} \exp \left(\pi \frac{M^{2}}{|q E|}\right) .
$$

This is much less restrictive than (64).

\subsection{Conventional contribution}

We are now going to calculate the temperature-dependent contributions to the mean current density and EMT, which are labelled by the upperscript $c$. We refer to these contributions as conventional contributions. Such contributions are not related to either particle creation or vacuum polarization. Thus, it is natural to say that they are determined only by the quantum statistical behavior of the initial thermal gas of charged particles in the constant external field under consideration.

\subsubsection{Proper-time representation}

Let us represent $\left\langle j_{\mu}(t)\right\rangle_{\theta}^{c}$ and $\left\langle T_{\mu \nu}(t)\right\rangle_{\theta}^{c}$ in (65) as integrals over the proper time. To this end, we relate $S_{\theta, \zeta}$ in (135) (see the Appendix) to the proper-time representation (55) of $S^{c}$; for details, see Subsec. 2 of the Appendix. For simplicity, we assume that the chemical potentials are not very large, $\left|\mu^{(\zeta)}\right|<M$. As always, we consider large $T$, namely,

$$
\sqrt{|q E|} T \gg 1+M^{2} /|q E| \text {. }
$$

Using (143), (145) and (147), as well as the explicit form (151) for $g\left(x, x^{\prime}, s, \tau\right)$, and calculating all the derivatives and traces, we find the following expressions for $\operatorname{Re}\left\langle j_{\mu}(t)\right\rangle_{\theta}^{c}$ and $\operatorname{Re}\left\langle T_{\mu \nu}(t)\right\rangle_{\theta}^{c}$ :

$$
\begin{aligned}
& \operatorname{Re}<j_{\mu}(t)>_{\theta}^{c}=\sum_{\zeta= \pm} \sum_{l=1}^{\infty}(-1)^{l+1} e^{\beta l \mu^{(\zeta)}} \operatorname{Re} J_{\mu}^{(l)}, \\
& J_{\mu}^{(l)}=\int_{0}^{\infty} d s \int_{0}^{\infty} d u h(s, u) Y(s, u) j_{\mu}(s, u), j_{\mu}(s, u)=i \delta_{\mu}^{3} q^{2} E(t+T / 2) \frac{\tau}{a-i \tau}, \\
& h(s, u)=-\frac{i}{8 \pi^{2} \sqrt{\pi}} \frac{q^{2} E B \cot [q B(s-i \tau)]}{u^{1 / 2} \sinh (q E s)}\left(\frac{a}{a-i \tau}\right)^{1 / 2} e^{-u / 4} e^{-M^{2}(i s+\tau)}, \\
& Y(s, u)=\exp \left[-(q E)^{2}(t+T / 2)^{2} \frac{\tau a}{a-i \tau}\right], \tau=(\beta l)^{2} / u,
\end{aligned}
$$

and

$$
\begin{aligned}
& \operatorname{Re}\left\langle T_{\mu \nu}(t)\right\rangle_{\theta}^{c}=\sum_{\zeta= \pm} \sum_{l=1}^{\infty}(-1)^{l+1} e^{\beta l \mu^{(\zeta)}} \operatorname{Re} T_{\mu \nu}^{(l)}, \\
& T_{\mu \nu}^{(l)}=\int_{0}^{\infty} d s \int_{0}^{\infty} d u h(s, u) Y(s, u) t_{\mu \nu}(s, u),
\end{aligned}
$$

where it is only the diagonal values $t_{\mu \nu}(s, u)$ that are nonzero:

$$
\begin{aligned}
t_{00}(s, u) & =\sum_{k=1,2,3} t_{k k}(s, u)+M^{2} \cosh (q E s), t_{11}(s, u)=t_{22}(s, u)=\frac{-i q B}{\sin [2 q B(s-i \tau)]} \\
t_{33}(s, u) & =\frac{-i}{2(a-i \tau) \cosh (q E s)}-\frac{(q E)^{2}(t+T / 2)^{2}}{\cosh (q E s)}\left(\frac{\tau}{a-i \tau}\right)^{2} .
\end{aligned}
$$


Note that the integrals over $s$ and $u$ in the representations (76) and (77) are finite. Such representations hold true for any $E$ and temperature for $t \in\left[t_{1}, t_{2}\right]$ within the restriction (75) on the interval $T$.

Representations (76) and (77) could be transformed to other forms, being more convenient for regions near $s=0$ in the integrands; for details, see Subsec. 3 of the Appendix. The result is that Re $J_{\mu}^{(l)}$ and $\operatorname{Re} T_{\mu \nu}^{(l)}$ in (76) and (77) can be presented as

$$
\operatorname{Re} J_{\mu}^{(l)}=\tilde{J}_{\mu}^{a}+\operatorname{Re} J_{\mu}^{b}, \operatorname{Re} T_{\mu \nu}^{(l)}=\tilde{T}_{\mu \nu}^{a}+\operatorname{Re} T_{\mu \nu}^{b},
$$

where

$$
\begin{aligned}
& \tilde{J}_{\mu}^{a}=\int_{0}^{\pi /|2 q E|-0} d s^{\prime} \int_{0}^{\infty} d u^{\prime} \tilde{h}\left(s^{\prime}, u^{\prime}\right) \tilde{Y}\left(s^{\prime}, u^{\prime}\right) \tilde{j}_{\mu}\left(s^{\prime}, u^{\prime}\right), \\
& \tilde{T}_{\mu \nu}^{a}=\int_{0}^{\pi /|2 q E|-0} d s^{\prime} \int_{0}^{\infty} d u^{\prime} \tilde{h}\left(s^{\prime}, u^{\prime}\right) \tilde{Y}\left(s^{\prime}, u^{\prime}\right) \tilde{t}_{\mu \iota}\left(s^{\prime}, u^{\prime}\right),
\end{aligned}
$$

and

$$
\begin{aligned}
& \tilde{h}\left(s^{\prime}, u^{\prime}\right)=-i h\left(i\left(c_{0}-s^{\prime}\right), u^{\prime}+u_{0}\right) \\
& =\frac{1}{8 \pi^{2} \sqrt{\pi}} \frac{q^{2} E B \operatorname{coth}\left[q B\left(s^{\prime}+\delta \tau\right)\right]}{u^{\prime 1 / 2} \sin \left(q E s^{\prime}\right)} \exp \left[-\frac{u^{\prime}+u_{0}}{4}-M^{2}\left(s^{\prime}+\delta \tau\right)\right], \\
& \tilde{Y}\left(s^{\prime}, u^{\prime}\right)=Y\left(i\left(c_{0}-s^{\prime}\right), u^{\prime}+u_{0}\right)=\exp \left[-\frac{(q E)^{2}(t+T / 2)^{2} \tau u^{\prime}}{\left(u^{\prime}+u_{0}\right)\left[1+(q E \tau)^{2}\right]}\right] \text {, } \\
& \delta \tau=\tau-(q E)^{-1} \arctan (q E \tau), \quad \tau=\frac{(\beta l)^{2}}{u^{\prime}+u_{0}} \\
& \tilde{j}_{\mu}\left(s^{\prime}, u^{\prime}\right)=j_{\mu}\left(i\left(c_{0}-s^{\prime}\right), u^{\prime}+u_{0}\right), \tilde{t}_{\mu \nu}\left(s^{\prime}, u^{\prime}\right)=t_{\mu \nu}\left(i\left(c_{0}-s^{\prime}\right), u^{\prime}+u_{0}\right), \\
& \tilde{j}_{\mu}\left(s^{\prime}, u^{\prime}\right)=-\delta_{\mu}^{3} \frac{q(q E)^{2}(t+T / 2) \tau\left[1+q E \tau \tan \left(q E s^{\prime}\right)\right]}{\tan \left(q E s^{\prime}\right)\left[1+(q E \tau)^{2}\right]}, \\
& \tilde{t}_{11}\left(s^{\prime}, u^{\prime}\right)=\tilde{t}_{22}\left(s^{\prime}, u^{\prime}\right)=\frac{q B}{\sinh \left[2 q B\left(s^{\prime}+\delta \tau\right)\right]} \text {, } \\
& \tilde{t}_{33}\left(s^{\prime}, u^{\prime}\right)=\frac{q E}{2 \sin \left(q E s^{\prime}\right)\left[1+(q E \tau)^{2}\right]^{1 / 2}}+\frac{(q E)^{4}(t+T / 2)^{2} \tau^{2}\left[1+q E \tau \tan \left(q E s^{\prime}\right)\right]}{\sin \left(q E s^{\prime}\right) \tan \left(q E s^{\prime}\right)\left[1+(q E \tau)^{2}\right]^{3 / 2}}, \\
& \tilde{t}_{00}\left(s^{\prime}, u^{\prime}\right)=\sum_{k=1,2,3} \tilde{t}_{k k}\left(s^{\prime}, u^{\prime}\right)+M^{2} \cos \left(q E s^{\prime}\right) \frac{1+q E \tau \tan \left(q E s^{\prime}\right)}{\left[1+(q E \tau)^{2}\right]^{1 / 2}} .
\end{aligned}
$$

Note that $\tau \leq(q E)^{-1} \tan \left(q E s^{\prime}\right)$ in $\tilde{J}_{\mu}^{a}$ and $\tilde{T}_{\mu \nu}^{a}$. The expressions for $J_{\mu}^{b}$ and $T_{\mu \nu}^{b}$ are given by (153) and (154), respectively. The form (79) is convenient for the study of both low-temperature approximations to a weak electric field and high-temperature approximations to an arbitrary electric field.

In a weak electric field, $|q E| / M^{2} \ll 1$, the quantities $\operatorname{Re} J_{\mu}^{b}$ and $\operatorname{Re} T_{\mu \nu}^{b}$ are exponentially small, so that in order to find the expansions of $\operatorname{Re} J_{\mu}^{(l)}$ and $\operatorname{Re} T_{\mu \nu}^{(l)}$ in the powers of $|q E| / M^{2}$, we use the relations

$$
\operatorname{Re} J_{\mu}^{(l)}=\tilde{J}_{\mu}^{a}, \quad \operatorname{Re} T_{\mu \nu}^{(l)}=\tilde{T}_{\mu \nu}^{a}
$$

where the exponentially small contributions have been neglected.

\subsubsection{Zero electric field}

When the electric field is zero, the mean current density is zero as well. Considering the limit $E \rightarrow 0$, one can see that the integral (80) over $s^{\prime}$ for $\tilde{T}_{\mu \nu}^{a}$ is taken from zero to infinity. In addition, the dependence on the variable $u^{\prime}$ in the kernel of the matrix elements $\tilde{T}_{\mu \nu}^{a}$ factors out as the multiplier $e^{-u^{\prime} / 4} / \sqrt{u^{\prime}}$. 
Calculating the integral over $u^{\prime}$ by using (139), in case $E=0$, we find

$$
\begin{aligned}
& \tilde{T}_{\mu \nu}^{a}=T_{\mu \nu}^{0}=\int_{0}^{\infty} d s \rho_{0}(s) t_{\mu \iota}^{0}(s) \\
& \rho_{0}(s)=\frac{q B \operatorname{coth}(q B s)}{4 \pi^{2} s} \exp \left[-M^{2} s-\frac{(\beta l)^{2}}{4 s}\right], t_{33}^{0}(s)=\frac{1}{2 s} \\
& t_{11}^{0}(s)=t_{22}^{0}(s)=\frac{q B}{\sinh (2 q B s)}, t_{00}^{0}(s)=\sum_{k=1,2,3} t_{k k}^{0}(s)+M^{2} .
\end{aligned}
$$

The quantities $T_{\mu \nu}^{0}$ are real-valued. Integrating by parts, one can see that $T_{00}^{0}$ has the form (84), where

$$
t_{00}^{0}(s)=-\frac{1}{2 s}+\frac{(\beta l)^{2}}{4 s^{2}} .
$$

In the case under consideration, the vacuum is stable, while the renormalized EMT (65) is real-valued, time-independent, and is given only by the conventional terms

$$
\begin{aligned}
\left.\left\langle T_{\mu \nu}(t)\right\rangle_{\text {ren }}\right|_{E=0} & =\left.\left\langle T_{\mu \nu}(t)\right\rangle_{r e n}^{c}\right|_{E=0}+\left.\left\langle T_{\mu \nu}(t)\right\rangle_{\theta}^{c}\right|_{E=0}, \\
\left.\left\langle T_{\mu \nu}(t)\right\rangle_{\theta}^{c}\right|_{E=0} & =\sum_{\zeta= \pm} \sum_{l=1}^{\infty}(-1)^{l+1} e^{\beta l \mu^{(\zeta)}} T_{\mu \nu}^{0},
\end{aligned}
$$

where $\left.\left\langle T_{\mu \nu}(t)\right\rangle_{r e n}^{c}\right|_{E=0}$ is determined by (59) at $E=0$.

The system under consideration is a relativistic fermionic gas in an external magnetic field, $B$, immersed in a sufficiently large quantization volume $V$. This system is described by the partition function

$$
Z=\operatorname{Tr} \exp \left\{\beta\left(\sum_{\zeta= \pm} \mu^{(\zeta)} N^{(\zeta)}-H\right)\right\},
$$

that follows from (15), $H=H\left(t_{1}\right)$, and by the thermodynamic potential $\Omega=-\theta \ln Z$. At the state of equilibrium, the temperature- and field-dependent effective Lagrangian $\mathcal{L}_{\text {ren }}^{\theta}$ is related to $\Omega$ as follows:

$$
\mathcal{L}_{\text {ren }}^{\theta}=-\Omega / V
$$

The mean values of energy density $\langle H\rangle / V$ and particle density $n^{(\zeta)}$ in the state (15) can be expressed in terms of $\mathcal{L}_{\text {ren }}^{\theta}$ as

$$
\begin{aligned}
\frac{\langle H\rangle}{V} & =-\mathcal{L}_{\text {ren }}^{\theta}-\beta \frac{\partial \mathcal{L}_{r e n}^{\theta}}{\partial \beta}+\sum_{\zeta= \pm} \mu^{(\zeta)} n^{(\zeta)} \\
n^{(\zeta)} & =\frac{\partial \mathcal{L}_{r e n}^{\theta}}{\partial \mu^{(\zeta)}} .
\end{aligned}
$$

Note that the energy density has been already calculated as a component of (85), namely,

$$
\left\langle H_{0}\right\rangle / V=\left.\left\langle T_{00}(t)\right\rangle_{r e n}\right|_{E=0} .
$$

Thus, we find that the effective Lagrangian has the form

$$
\begin{aligned}
\mathcal{L}_{\text {ren }}^{\theta} & =\left.\mathcal{L}_{\text {ren }}\right|_{E=0}+\Delta \mathcal{L}_{\theta}, \\
\Delta \mathcal{L}_{\theta} & =\frac{1}{8 \pi^{2}} \sum_{\zeta= \pm} \sum_{l=1}^{\infty}(-1)^{l+1} e^{\beta l \mu^{(\zeta)}} \int_{0}^{\infty} \frac{d s}{s^{2}} \exp \left[-M^{2} s-\frac{(\beta l)^{2}}{4 s}\right] q B \operatorname{coth}(q B s)
\end{aligned}
$$

where $\left.\mathcal{L}_{r e n}\right|_{E=0}$ is the renormalized Heisenberg-Euler effective Lagrangian (58) at $E=0$.

It turns out that the space components of $\left.\left\langle T_{i k}(t)\right\rangle_{r e n}\right|_{E=0}$ (85) can be derived from the effective Lagrangian $\mathcal{L}_{\text {ren }}^{\theta}$ as follows:

$$
\begin{aligned}
\left.\left\langle T_{11}(t)\right\rangle_{r e n}\right|_{E=0} & =\left.\left\langle T_{22}(t)\right\rangle_{r e n}\right|_{E=0}=\mathcal{L}_{r e n}^{\theta}-B \frac{\partial \mathcal{L}_{r e n}^{\theta}}{\partial B}, \\
\left.\left\langle T_{33}(t)\right\rangle_{r e n}\right|_{E=0} & =\mathcal{L}_{r e n}^{\theta} .
\end{aligned}
$$


It should be noted that the finite temperature and density effects are contained only in $\Delta \mathcal{L}_{\theta}$. In particular, the thermodynamic potential $\Omega_{\text {free }}$ of perfect free Fermi gas at $B=0$ is related to $\left.\Delta \mathcal{L}_{\theta}\right|_{B=0}$ in the form $\Omega_{\text {free }}=-\left.\Delta \mathcal{L}_{\theta}\right|_{B=0} V$. Expressing the proper-time integral for $\left.\Delta \mathcal{L}_{\theta}\right|_{B=0}$ in terms of the modified Bessel function of the third kind (Macdonald's function) $K_{\nu}(z)$ [34, and using formula 3.471 .9 in [40],

$$
\int_{0}^{\infty} x^{\nu-1} e^{-a / x-b x} d x=2\left(\frac{a}{b}\right)^{\nu / 2} K_{\nu}(2 \sqrt{a b})
$$

we find

$$
\left.\Delta \mathcal{L}_{\theta}\right|_{B=0}=\left(\frac{M}{\beta \pi}\right)^{2} \sum_{\zeta= \pm} \sum_{l=1}^{\infty} \frac{(-1)^{l+1}}{l^{2}} e^{\beta l \mu^{(\zeta)}} K_{2}(M \beta l) .
$$

Thus, we obtain for the quantity $\Omega_{\text {free }}$ the expression well-known from textbooks.

Considering the usual case $\mu^{(+)}=-\mu^{(-)}=\mu$, we can see that (89) is identical with the results obtained earlier in the case $E=0$ : see Eq. (52) in the paper [15, where the imaginary-time formalism was used, and Eq. (5.20) in [16], where the real-time formalism was used (we disregard the factor 1/2 in Eq. (5.20) of [16] as an misprint). In the case $\mu=0$, Eq. (89) is in agreement with the result obtained in 14 .

It should be noted that our analysis deals primarily with the effects of electric field at finite initial temperatures, and therefore we do not examine in detail the particular case of magnetic field (at $E=0)$. Note, however, that the leading magnetic field-dependent terms follow immediately from (89). We present these terms for comparison with a nonzero electric field: in the weak-field limit, when either $|q B| / M^{2} \ll 1$ or $|q B| \beta^{2} \ll 1$, we have

$$
\mathcal{L}_{1}=\Delta \mathcal{L}_{\theta}-\left.\Delta \mathcal{L}_{\theta}\right|_{B=0}=\frac{(q B)^{2}}{12 \pi^{2}} \sum_{\zeta= \pm} \sum_{l=1}^{\infty}(-1)^{l+1} e^{\beta l \mu^{(\zeta)}} K_{0}(M \beta l)
$$

In the strong-field limit, $|q B| \gg M^{2}, \beta^{-2}$, we have

$$
\mathcal{L}_{1}=\frac{|q B| M}{2 \pi^{2} \beta} \sum_{\zeta= \pm} \sum_{l=1}^{\infty} \frac{(-1)^{l+1}}{l} e^{\beta l \mu^{(\zeta)}} K_{1}(M \beta l) .
$$

The latter quantity is small in comparison with the corresponding vacuum polarization term, which is given by the following expression:

$$
\left.\mathcal{L}_{r e n}\right|_{E=0}-\left.\mathcal{L}_{r e n}\right|_{E=0, B=0}=\frac{(q B)^{2}}{24 \pi^{2}} \ln \frac{|q B|}{M^{2}} .
$$

In the weak-field limit, we can see that the leading field-dependent thermal contributions to EMT have the form

$$
\begin{aligned}
\tau_{11}^{B} & =\tau_{22}^{B}=\mathcal{L}_{1}-B \frac{\partial \mathcal{L}_{1}}{\partial B}=-\mathcal{L}_{1}, \quad \tau_{33}^{B}=\mathcal{L}_{1} \\
\tau_{00}^{B} & =-\mathcal{L}_{1}-\beta \frac{\partial \mathcal{L}_{1}}{\partial \beta}+\sum_{\zeta= \pm} \mu^{(\zeta)} \frac{\partial \mathcal{L}_{1}}{\partial \mu^{(\zeta)}}
\end{aligned}
$$

Note that Fermi systems with an external magnetic field have been under intensive study, and various asymptotic expansions of the effective Lagrangian at finite temperatures have been made: for a review, see [15, 16]; a review on finite-temperature free relativistic systems can be found, e.g., in [32, 41, 42].

\subsubsection{Weak electric field and low temperatures}

In a weak electric field, $|q E| / M^{2} \ll 1$, the quantities $\operatorname{Re} J_{\mu}^{(l)}$ and $\operatorname{Re} T_{\mu \nu}^{(l)}$ in (76) and (177) are given by $\tilde{J}_{\mu}^{a}$ and $\tilde{T}_{\mu \nu}^{a}$ (80)- (82); see (83). The dependence of expressions (80)- 82 on the parameters $E$ and $\beta$ is described by $q E \tau$, which is a function of two variables: $s^{\prime}$ and $u^{\prime}$. Approximations of this function and of the corresponding integral over $u^{\prime}$ at low temperatures and at high temperatures are quite different. Therefore, unlike the case $E=0$, these limiting cases must be studied separately. In this subsection, we shall examine the case of low temperatures, $M \beta \gg 1$.

Contributions to integrals (80), given by the region $M^{2} s^{\prime} \gg 1$ of large $s^{\prime}$, are exponentially small. Therefore, we limit the range of integration over $s^{\prime}$ from above by the value $\chi / M^{2}$, so that $1 \ll \chi \ll$ $M^{2} /|q E|$. At low temperatures, the leading contributions to the integral over $s^{\prime}$ are formed at the region $M^{2} s^{\prime} \sim M \beta l / 2 \gg 1$, provided that $M \beta l / 2 \ll \chi$. It is interesting to examine an approximation which 
admits the limiting process $E \rightarrow 0$. For every $l \leq l_{\max }$ in a sufficiently weak electric field, there exists such $\chi$ that $M \beta l_{\max } / 2 \ll \chi$. Under this condition, we obtain the approximation for finite values of $l$. Note that at low temperatures the terms $e^{\mu^{(\zeta)} \beta l} \operatorname{Re} J_{\mu}^{(l)}$ and $e^{\mu^{(\zeta)} \beta l} \operatorname{Re} T_{\mu \nu}^{(l)}$ of order $e^{-\left(M-\mu^{(\zeta)}\right) \beta l} l^{-\alpha}$ in case $\alpha \geq 1 / 2$ decrease when $l$ increases. Therefore, any accuracy of calculation for the sums in (76) and (77) can be provided by a finite number of summands. Accordingly, at $\left|\mu^{(\zeta)}\right| \ll M$, in order to estimate the leading contributions it is sufficient to take into account only the first summand, at $l=1$. However, even for large values of chemical potentials, $\left|\mu^{(\zeta)}\right| \sim M$, a sufficient accuracy is provided by summation up to a corresponding finite number $l_{\text {est }}$. Accordingly, we suppose that $l_{\text {est }} \leq l_{\max }$. Thus, the approximated expressions for $\operatorname{Re}\left\langle j_{\mu}(t)\right\rangle_{\theta}^{c}$ and $\operatorname{Re}\left\langle T_{\mu \nu}(t)\right\rangle_{\theta}^{c}$ at low temperatures have the form

$$
\begin{aligned}
& \operatorname{Re}\left\langle j_{\mu}(t)\right\rangle_{\theta}^{c}=\sum_{\zeta= \pm} \sum_{l=1}^{l_{e s t}}(-1)^{l+1} e^{\beta l \mu^{(\zeta)}} J_{\mu}^{\chi}, \\
& J_{\mu}^{\chi}=\int_{0}^{\chi / M^{2}} d s \int_{0}^{\infty} d u \tilde{h}(s, u) \tilde{Y}(s, u) \tilde{j}_{\mu}(s, u) ; \\
& \operatorname{Re}\left\langle T_{\mu \nu}(t)\right\rangle_{\theta}^{c}=\sum_{\zeta= \pm} \sum_{l=1}^{l_{e s t}}(-1)^{l+1} e^{\beta l \mu^{(\zeta)}} T_{\mu \nu}^{\chi}, \\
& T_{\mu \nu}^{\chi}=\int_{0}^{\chi / M^{2}} d s \int_{0}^{\infty} d u \tilde{h}(s, u) \tilde{Y}(s, u) \tilde{t}_{\mu \iota}(s, u),
\end{aligned}
$$

where $\tilde{h}(s, u), \tilde{Y}(s, u), \tilde{j}_{\mu}(s, u)$, and $\tilde{t}_{\mu \iota}(s, u)$ are defined in (81) and (82).

Let us find the leading contributions depending on the electric field for expressions (95). These quantities depend on two dimensionless parameters that characterize the influence of electric field: $|q E| / M^{2}$ is a parameter determined by the strength of constant electric field, whereas $q E(t+T / 2) / M$ is a parameter depending on time $t$ and determined by the increment in the kinetic momentum of a particle in electric field by the time instant $t$ (i.e., the increment that accumulates during the time interval $t-t_{1}=t+T / 2$ ). The first parameter $|q E| / M^{2}$ is small in a weak electric field. We shall expand all the functions in the integrals (95) as series in the powers of this parameter and examine only the leading terms of the expansion. In addition, the value $|q E|(t+T / 2) / M$ is not necessarily small for a sufficiently large time interval, $t+T / 2$. Thus, the dependence on this value in the following expression is taken into account precisely. For simplicity, we assume that the magnetic field is weak, so we can neglect the biquadratic terms of the order $q^{2}|E B| / M^{4}$. Therefore, with accuracy up to the leading term depending on the electric field, we obtain

$$
\begin{aligned}
J_{\mu}^{\chi} & =\int_{0}^{\infty} d s \int_{0}^{\infty} d u h^{0} Y^{W} j_{\mu}^{W}, \\
T_{\mu \nu}^{\chi} & =\int_{0}^{\infty} d s \int_{0}^{\infty} d u\left(h^{0}+\delta h\right) Y^{W}\left(t_{\mu \iota}^{0}+\delta t_{\mu \iota}\right) .
\end{aligned}
$$

Here, we have neglected the exponentially small contribution and extended the range of integration over $s$ to infinity, $+\infty$; besides,

$$
\begin{aligned}
& h^{0}=\frac{e^{-u / 4}}{2 \sqrt{\pi u}} \rho_{0}, \quad \delta h=\frac{e^{-u / 4}}{2 \sqrt{\pi u}} \frac{(q E)^{2}}{4 \pi^{2}} \exp \left[-M^{2} s-\frac{(\beta l)^{2}}{4 s}\right] D, \\
& D=\left[\frac{(\beta l)^{2}}{12 s}-\frac{\tau_{W}^{3}}{3 s^{2}}\left(\frac{1}{s}+M^{2}\right)+\frac{1}{6}\right], \quad Y^{W}=\exp \left[-\frac{(q E)^{2}(t+T / 2)^{2} \tau_{W} u}{u+(\beta l)^{2} / s}\right], \\
& \tau_{W}=\frac{(\beta l)^{2}}{u+(\beta l)^{2} / s}, \quad j_{\mu}^{W}=-\delta_{\mu}^{3} q^{2} E(t+T / 2) \tau_{W} / s, \delta t_{11}=\delta t_{22}=-\frac{(q E)^{2} \tau_{W}^{3}}{6 s^{2}}, \\
& \delta t_{33}=\frac{(q E)^{2}}{4}\left(\frac{s}{3}-\frac{\tau_{W}^{2}}{s}\right)+(q E)^{2}(t+T / 2)^{2}\left(\frac{\tau_{W}}{s}\right)^{2}, \\
& \delta t_{00}=\sum_{k=1,2,3} \delta t_{k k}+\delta t_{M}, \quad \delta t_{M}=-\frac{1}{2} M^{2}(q E)^{2}\left(s-\tau_{W}\right)^{2},
\end{aligned}
$$

where $t_{\mu \iota}^{0}$ and $\rho_{0}$ are given by (84). Note that in expressions (97) we have not imposed any conditions on temperature. Let us do it now. Namely, since at low temperatures the leading contributions to the 
integral over $s$ are formed at the region of $s \sim \beta l /(2 M)$, the function $\tau_{W}$ can be expanded as a series in the powers of $u$,

$$
\tau_{W}=s\left\{1-\frac{s u}{(\beta l)^{2}}+\left[\frac{s u}{(\beta l)^{2}}\right]^{2}-\cdots\right\} .
$$

We consider only the leading contribution to the expansion in inverse powers of $\beta M$. To calculate this contribution, it is sufficient to take into account the first three terms of expansion (98); moreover, in the coefficients at time-dependent functions it is sufficient to take into account only the first term of the expansion. Therefore, at low temperatures the function $Y^{W}$ in the leading approximation has the form

$$
Y^{W}=Y^{W L}=\exp \left[-(q E)^{2}(t+T / 2)^{2} \frac{s^{2} u}{(\beta l)^{2}}\right] .
$$

Using expansion (98) and expression (99), we can calculate the integrals over $u$ in (96). To this end, it is convenient to use the generating function $\Phi_{0}(\alpha)$, defined by the integral

$$
\Phi_{0}(\alpha)=\frac{1}{2 \sqrt{\pi}} \int_{0}^{\infty} \frac{d u}{u^{1 / 2}} e^{-u(1 / 4+\alpha)} .
$$

Calculating this integral according to formula (139), we obtain $\Phi_{0}(\alpha)=(1+4 \alpha)^{-1 / 2}$. Then, taking derivatives with respect to the parameter $\alpha$, we obtain a formula we need for calculation:

$$
\Phi_{n}(\alpha)=\frac{1}{2 \sqrt{\pi}} \int_{0}^{\infty} \frac{d u}{u^{1 / 2}} e^{-u(1 / 4+\alpha)} u^{n}=(-1)^{n} \frac{\partial^{n} \Phi_{0}(\alpha)}{\partial \alpha^{n}} .
$$

In the case under consideration, we have $\alpha=(q E)^{2}(t+T / 2)^{2}\left(\frac{s}{\beta l}\right)^{2}$.

Until the $T$-constant field has switched on, the system in question is at the state of thermal equilibrium. When the $T$-constant field switches on at the instant $t_{1}$ the particles at the initial state begin to accelerate. The corresponding current density and EMT change with time until the moment $t_{2}$, when the $T$-constant field turns off. Nevertheless, such a non-equilibrium state during a certain interval of time until the increment of kinetic momentum is sufficiently small, $|q E|(t+T / 2) / M \ll 1$, is not very different from the initial state of thermal equilibrium, and it makes sense to compare these states. It is exactly this condition that guarantees the existence of a limiting process $E \rightarrow 0$. Therefore, it is important to examine such a case in more detail. On the other hand, it is of special interest to examine the case of asymptotically large values of time, when $|q E|(t+T / 2) / M \gg 1$. In this case, the presence of electric field manifests itself to the maximum. In what follows, we examine two limiting cases.

Small increment of kinetic momentum When the increment of kinetic momentum is small, $|q E|(t+T / 2) / M \ll$ 1 , the kernels in (96) can be expanded in its powers. To this end, we take into account the fact that $\alpha=0$ implies that the function $\Phi_{n}(\alpha)$ is expressed in terms of binomial coefficients, $\Phi_{0}(0)=1$ and $\Phi_{n}(0)=2^{n} \cdot 1 \cdot 3 \cdot 5 \cdot \ldots \cdot(2 n-1)$, for $n \geq 1$. Using (96) after integrating over $u$, and considering only the leading time-dependent contributions, we obtain

$$
J_{\mu}^{\chi}=j_{\mu}^{0} \int_{0}^{\infty} d s \rho_{0}, T_{\mu \nu}^{\chi}=T_{\mu \nu}^{0}+\Delta T_{\mu \nu},
$$

where $T_{\mu \nu}^{0}$ and $\rho_{0}$ are independent of electric field and given by (84); besides

$$
j_{\mu}^{0}=-\delta_{\mu}^{3} q^{2} E(t+T / 2)
$$


In the above representation of $T_{\mu \nu}^{\chi}$, the part that depends on the electric field is separated as $\Delta T_{\mu \nu}$ and has the form

$$
\begin{aligned}
\Delta T_{\mu \nu} & =\frac{1}{4 \pi^{2}} \int_{0}^{\infty} d s \exp \left[-M^{2} s-\frac{(\beta l)^{2}}{4 s}\right] \Delta t_{\mu \nu} \\
\Delta t_{11} & =\Delta t_{22}=\frac{(q E)^{2}}{2 s}\left(D_{0}-\frac{1}{3}\right)-\frac{(q E)^{2}(t+T / 2)^{2}}{s(\beta l)^{2}} \\
\Delta t_{33} & =\Delta t_{11}+\frac{(q E)^{2}}{2(\beta l)^{2}}+\frac{(q E)^{2}(t+T / 2)^{2}}{s^{2}} \\
\Delta t_{00} & =(q E)^{2} M^{2} D_{0}+\frac{(q E)^{2}(t+T / 2)^{2}}{(\beta l)^{2}}\left[\frac{(\beta l)^{2}}{s^{2}}-2 M^{2}\right] \\
D_{0} & =-\frac{1}{6}+\frac{(\beta l)^{2}}{12 s}-\frac{M^{2} s}{3}+2 \frac{M^{2} s^{2}}{(\beta l)^{2}}-24 \frac{M^{2} s^{3}}{(\beta l)^{4}}
\end{aligned}
$$

Integration in (104) leads to the modified Bessel functions of the third kind $K_{\nu}(z)$, according to formula (91). For simplicity, we assume that the chemical potentials are such that $e^{-\left(M-\mu^{(\zeta)}\right) \beta} \ll 1$. We then use the asymptotic expansion 7.13.1.(7) [34:

$$
K_{\nu}(z)=\left(\frac{\pi}{2 z}\right)^{1 / 2} e^{-z}\left[1+\left(\nu^{2}-\frac{1}{4}\right) \frac{1}{2 z}+\left(\nu^{2}-\frac{1}{4}\right)\left(\nu^{2}-\frac{9}{4}\right) \frac{1}{8 z^{2}}+O\left(|z|^{-3}\right)\right] .
$$

As a result of the asymptotic expansion at low temperatures, we obtain the leading contributions of $\Delta T_{\mu \nu}$ in the form

$$
\begin{aligned}
\Delta T_{11} & =\Delta T_{22}=-C_{l} \frac{2}{M \beta l}\left[1+\frac{M(t+T / 2)^{2}}{\beta l}\right]\left[1+O\left(\frac{1}{M \beta l}\right)\right], \\
\Delta T_{33} & =C_{l}\left[-\frac{3}{2 M \beta l}+4 \frac{M(t+T / 2)^{2}}{\beta l}\right]\left[1+O\left(\frac{1}{M \beta l}\right)\right], \\
\Delta T_{00} & =C_{l}\left[-\frac{235}{256}+2 \frac{M(t+T / 2)^{2}}{\beta l}\right]\left[1+O\left(\frac{1}{M \beta l}\right)\right], \\
C_{l} & =\frac{(q E)^{2}}{4 \pi^{2}}\left(\frac{\pi}{2 M \beta l}\right)^{1 / 2} e^{-M \beta l} .
\end{aligned}
$$

This makes it possible to present the expression for EMT $\operatorname{Re}\left\langle T_{\mu \nu}(t)\right\rangle_{\theta}^{c}$ in (95) as follows:

$$
\operatorname{Re}\left\langle T_{\mu \nu}(t)\right\rangle_{\theta}^{c}=\left.\left\langle T_{\mu \nu}(t)\right\rangle_{\theta}^{c}\right|_{E=0}+\sum_{\zeta= \pm} \sum_{l=1}^{l_{e s t}}(-1)^{l+1} e^{\beta l \mu^{(\zeta)}} \Delta T_{\mu \nu}
$$

where we have explicitly separated the part $\left.\left\langle T_{\mu \nu}(t)\right\rangle_{\theta}^{c}\right|_{E=0}$, independent of the electric field, which is defined in (85), while the terms $\Delta T_{\mu \nu}$ depending on the electric field are given by (106).

With the help of the expression for $J_{\mu}^{\chi}$ in (102), the current density $\operatorname{Re}\left\langle j_{\mu}(t)\right\rangle_{\theta}^{c}$ in (95), with the corresponding accuracy, can be expressed in the form

$$
\operatorname{Re}\left\langle j_{\mu}(t)\right\rangle_{\theta}^{c}=-\delta_{\mu}^{3} \frac{q^{2} E(t+T / 2)}{M} \sum_{\zeta= \pm} n^{(\zeta)}
$$

where the initial particle density $n^{(\zeta)}$ is given by (88). Considering only the leading asymptotic contributions, we obtain $n^{(\zeta)}$ as follows:

$$
n^{(\zeta)}=2\left(\frac{M}{2 \pi \beta}\right)^{3 / 2} \sum_{l=1}^{l_{\text {est }}}(-1)^{l+1} e^{\beta l\left(\mu^{(\zeta)}-M\right)} \frac{1}{l^{3 / 2}} \frac{q B \beta l}{2 M} \operatorname{coth} \frac{q B \beta l}{2 M} .
$$

If the chemical potential is small, $\left|\mu^{(\zeta)}\right| \ll M$, the leading contribution in the sums (107), (109) yields one term, at $l=1$. In this case, the leading magnetic field-dependent thermal contributions (94) in $\operatorname{Re}\left\langle T_{\mu \nu}(t)\right\rangle_{\theta}^{c}$ are $\tau_{11}^{B}=\tau_{22}^{B}=-\tau_{33}^{B}<0, \tau_{00}^{B}>0$, and all the time-independent contributions for electric field-dependent terms in (107) are negative. 
Large increment of kinetic momentum When the increment of kinetic momentum is large, $|q E|(t+T / 2) / M \gg$ 1 , the parameter $\alpha$ in (100) is large as well. Then, we calculate the integrals over $u$ in (96), by using the asymptotics of $\Phi_{0}(\alpha), \Phi_{0}(\alpha) \simeq \alpha^{-1 / 2} / 2$, and obtain

$$
\begin{aligned}
J_{\mu}^{\chi} & =\frac{j_{\mu}^{0} \beta l}{2|q E|(t+T / 2)} \int_{0}^{\infty} d s \rho_{0} s^{-1}, \\
T_{\mu \nu}^{\chi} & =\frac{\beta l}{2|q E|(t+T / 2)} \int_{0}^{\infty} d s \rho_{0} s^{-1}\left(t_{\mu \iota}^{0}+\left.\delta t_{\mu \iota}\right|_{\tau_{W}=s}\right),
\end{aligned}
$$

where we have used only the first term of expansion (98). At low temperatures, the main contribution to the integral over $s$ is formed at the region $s \sim \beta l /(2 M)$, and thus the leading contributions in (110) can be found immediately:

$$
\begin{aligned}
J_{\mu}^{\chi} & =\frac{j_{\mu}^{0} M}{|q E|(t+T / 2)} \int_{0}^{\infty} d s \rho_{0}, \\
T_{\mu \nu}^{\chi} & =\frac{M}{|q E|(t+T / 2)} \int_{0}^{\infty} d s \rho_{0}\left(t_{\mu \iota}^{0}+\delta^{0} t_{\mu \iota}\right),
\end{aligned}
$$

where

$$
\delta^{0} t_{11}=\delta^{0} t_{22}=0, \quad \delta^{0} t_{00}=\delta^{0} t_{33}=(q E)^{2}(t+T / 2)^{2} .
$$

Consequently, using expressions (111) we obtain from representation (95) that

$$
\begin{aligned}
\operatorname{Re}\left\langle j_{\mu}(t)\right\rangle_{\theta}^{c} & =-\delta_{\mu}^{3}|q| \operatorname{sgn}(E) \sum_{\zeta= \pm} n^{(\zeta)}, \\
\operatorname{Re}\left\langle T_{11}(t)\right\rangle_{\theta}^{c} & =\operatorname{Re}\left\langle T_{22}(t)\right\rangle_{\theta}^{c}=\left.\frac{M}{|q E|(t+T / 2)} \operatorname{Re}\left\langle T_{11}(t)\right\rangle_{\theta}^{c}\right|_{E=0}, \\
\operatorname{Re}\left\langle T_{00}(t)\right\rangle_{\theta}^{c} & =\operatorname{Re}\left\langle T_{33}(t)\right\rangle_{\theta}^{c}=|q E|(t+T / 2) \sum_{\zeta= \pm} n^{(\zeta)},
\end{aligned}
$$

where $n^{(\zeta)}$ is the initial particle density, given by (109), and $\left.\operatorname{Re}\left\langle T_{11}(t)\right\rangle_{\theta}^{c}\right|_{E=0}$ is the initial value of $\operatorname{Re}\left\langle T_{11}(t)\right\rangle_{\theta}^{c}$, defined by (85) at $t<t_{1}$.

\subsubsection{High temperatures}

Let us find the leading field-dependent contributions for $\operatorname{Re}\left\langle j_{\mu}(t)\right\rangle_{\theta}^{c}$ and $\operatorname{Re}\left\langle T_{\mu \nu}(t)\right\rangle_{\theta}^{c}$ at high temperatures. In the presence of external magnetic and electric fields, the conditions of the applicability of high-temperature asymptotic include the following conditions for the field strengths:

$$
M \beta \ll 1, \quad \sqrt{|q B|} \beta \ll 1, \quad \sqrt{|q E|} \beta \ll 1 .
$$

In the case of fields that are not very strong, $|q B| / M^{2} \leq 1,|q E| / M^{2} \leq 1$, these subsidiary conditions are not relevant; however, they become necessary in the case of strong fields, $|q B| / M^{2}>1,|q E| / M^{2}>1$. There are no other necessary restrictions for the field strengths. Under these restrictions, it is convenient to use the representation (79). At high temperatures, the leading contributions into the expressions for $\operatorname{Re} J_{\mu}^{(l)}$ and $\operatorname{Re} T_{\mu \nu}^{(l)}$ are given by the terms $\tilde{J}_{\mu}^{a}$ and $\tilde{T}_{\mu \nu}^{a}$, respectively. These terms emerge from the integrals (80). In turn, at high temperatures, the leading contributions to these integrals emerge from small values of $s^{\prime}$, namely, $s^{\prime} \sim(\beta l)^{2}$. Therefore, in calculating the leading contributions to all the field-dependent functions one can assume that $|q B| s^{\prime} \ll 1$ and $|q E| s^{\prime} \ll 1$. This allows one to use, in the integrals (80), expansions in the powers of $|q B| s^{\prime}$ and $|q E| s^{\prime}$, and then to extend the range of integration over $s^{\prime}$ to $+\infty$, while neglecting the exponentially small contribution. As a result, one arrives at the conclusion that $\tilde{J}_{\mu}^{a}$ and $\tilde{T}_{\mu \nu}^{a}$, are approximated by the expressions $J_{\mu}^{\chi}$ and $T_{\mu \nu}^{\chi}$ in (96), respectively. Note that at high temperatures the leading contribution into the sums (76) and (77) is formed by a finite number of summands, when $l$ belongs to the range $1 \leq l \leq l_{\max }$. A choice for the number $l_{\max }$ is determined by a given accuracy of calculation, and by the fact that $l_{\max } \ll(\sqrt{|q E|} \beta)^{-1}$ and $l_{\max } \ll(\sqrt{|q B|} \beta)^{-1}$. The terms (96) are well-decreasing functions of the number $l$, and therefore in the high-temperature limit one can assume that $l_{\max } \rightarrow \infty$. Thus, the sum is taken over positive integers, as in the case of thermal equilibrium, which happens in the absence of electric field. As a result, we obtain the following 
asymptotic expressions for $\operatorname{Re}\left\langle j_{\mu}(t)\right\rangle_{\theta}^{c}$ and $\operatorname{Re}\left\langle T_{\mu \nu}(t)\right\rangle_{\theta}^{c}$ :

$$
\begin{aligned}
\operatorname{Re}\left\langle j_{\mu}(t)\right\rangle_{\theta}^{c} & =\sum_{\zeta= \pm} \sum_{l=1}^{\infty}(-1)^{l+1} e^{\beta l \mu^{(\zeta)}} J_{\mu}^{\chi}, \\
\operatorname{Re}\left\langle T_{\mu \nu}(t)\right\rangle_{\theta}^{c} & =\sum_{\zeta= \pm} \sum_{l=1}^{\infty}(-1)^{l+1} e^{\beta l \mu^{(\zeta)}} T_{\mu \nu}^{\chi},
\end{aligned}
$$

where $J_{\mu}^{\chi}$ and $T_{\mu \nu}^{\chi}$ are given by expressions (96) and (97). As usual, in these asymptotics, we suppose $\left|\mu^{(\zeta)}\right| \beta \ll 1$.

As a whole, we are interested in the leading electric-field-dependent contributions into expressions (114), as well as in the limiting process $E \rightarrow 0$, and in the possibility to compare the leading electricfield-dependent term with the leading magnetic-field-dependent term. Therefore, we assume for simplicity that the magnetic field is not stronger than the electric field, $|B / E| \leq 1$; then, within the given accuracy of calculation, we can neglect the contributions that contain the magnetic field at powers higher than the second, as well as the contributions depending on the product $B^{2} E^{2}$.

At high temperatures, the dimensionless parameter that depends on time $(t)$ and characterizes the influence of electric field has the form $q E(t+T / 2) \beta$. As in the low-temperature case, this parameter is determined by the relative increment of the kinetic momentum of a particle in electric field reached by the time instant $t$; however, the scale on which one estimates this increment is now the temperature $\theta$ rather than the mass $M$.

When the $T$-constant field switches on, the particles found at the initial state begin to accelerate. Nevertheless, such a non-equilibrium state is not very different from the initial state of thermal equilibrium when $|q E|(t+T / 2) \beta \ll 1$, and it makes sense to compare these states. It is exactly this condition that guarantees the existence of the limiting process $E \rightarrow 0$. It is clear that at any finite value $|q E|(t+T / 2)$ the given condition takes place when $\beta \rightarrow 0$, i.e., it is exactly in this case that we deal with an asymptotic expression at high temperatures. Therefore, this case is the most interesting one. Let us note, in addition, that this condition implies comparatively weaker restrictions for the value of kinetic momenta, $|q E|(t+T / 2) \ll 1 / \beta$ than the values at low temperatures when one requires that $|q E|(t+T / 2) \ll M$. For sufficiently high temperatures, ultrarelativistic kinetic momenta can be regarded as sufficiently small. The opposite case of asymptotically large values of time at large but finite $\beta$, when $|q E|(t+T / 2) \beta \gg 1$, is a complicated case, when, according to conditions (113), the temperature is sufficiently high in comparison with $M, B$, and $E$; however, at the same time, the temperature is low in comparison with the kinetic momentum $q E(t+T / 2)$. This case is also interesting, since the presence of electric field manifests itself to the maximum. In what follows, we examine these two limiting cases.

Small increment of kinetic momentum When the increment of kinetic momentum is small, $|q E|(t+T / 2) \beta \ll$ 1, the function $Y^{W}$ in (97) can be expanded as

$$
Y^{W}=1-\delta Y, \quad \delta Y=(q E)^{2}(t+T / 2)^{2}\left(\tau_{W}-s^{-1} \tau_{W}^{2}\right)
$$

Let us examine the following approximation:

$$
\begin{aligned}
J_{\mu}^{\chi} & =\left.j_{\mu}^{0} \int_{0}^{\infty} \frac{d s}{s} \int_{0}^{\infty} d u h^{0}\right|_{B=0} \tau_{W}, \\
T_{\mu \nu}^{\chi} & =T_{\mu \nu}^{0}+\Delta T_{\mu \nu},
\end{aligned}
$$

where $T_{\mu \nu}^{0}$ and $h^{0}$ are expressions being independent of electric field; $T_{\mu \nu}^{0}$ is given by (84); $h^{0}$ and $\tau_{W}$ are given by (97); $j_{\mu}^{0}$ is given by (103); besides

$$
\begin{aligned}
\Delta T_{\mu \nu} & =\frac{1}{4 \pi^{2}} \int_{0}^{\infty} d s \exp \left[-M^{2} s-\frac{(\beta l)^{2}}{4 s}\right]\left(\Delta t_{\mu \nu}^{1}+\Delta t_{\mu \nu}^{2}\right) \\
\Delta t_{\mu \nu}^{1} & =\left.\frac{1}{2 \sqrt{\pi}} \int_{0}^{\infty} \frac{d u}{u^{1 / 2}} e^{-u / 4} \frac{1}{s^{2}} \delta t_{\mu \nu}\right|_{B=0} \\
\Delta t_{\mu \nu}^{2} & =\left.\frac{1}{2 \sqrt{\pi}} \int_{0}^{\infty} \frac{d u}{u^{1 / 2}} e^{-u / 4}\left[(q E)^{2} D-\delta Y \frac{1}{s^{2}}\right] t_{\mu \nu}^{0}\right|_{B=0}
\end{aligned}
$$

where $t_{\mu \nu}^{0}$ is given by (84), $\delta t_{\mu \nu}$ and $D$ are given by (97), respectively.

To calculate the integrals in (115) and (116), we need the following functions of a real argument $p$ :

$$
I_{n}(p)=\frac{1}{2 \sqrt{\pi}} \int_{0}^{\infty} \frac{d u}{u^{1 / 2}} e^{-u / 4} \frac{1}{(u+p)^{n}}, \quad n \geq 1 .
$$


By using formula (3.363.2) in [40, the function $I_{1}(p)$ can be expressed in terms of the incomplete gammafunction:

$$
I_{1}(p)=\frac{1}{2 p^{1 / 2}} e^{p / 4} \Gamma\left(\frac{1}{2}, \frac{p}{4}\right) .
$$

Differentiating this relation with respect to the variable $p$, one can find the corresponding representation for the remaining functions $I_{n}(p)$ at $n \geq 2$. In particular, we have

$$
I_{2}(p)=\frac{1}{2}\left(\frac{1}{p}-\frac{1}{2}\right) I_{1}(p)+\frac{1}{4 p} .
$$

In the case under consideration, $p=(\beta l)^{2} / s$. Let us express $J_{\mu}^{\chi}$ and $\Delta t_{k k}$ for $k=1,2,3$ in terms of the functions $I_{n}(p)$ :

$$
\begin{aligned}
& J_{\mu}^{\chi}=\frac{j_{\mu}^{0}(\beta l)^{2}}{4 \pi^{2}} \int_{0}^{\infty} \frac{d s}{s^{3}} \exp \left[-M^{2} s-\frac{(\beta l)^{2}}{4 s}\right] I_{1}\left(\frac{(\beta l)^{2}}{s}\right), \\
& \Delta t_{11}^{1}=\Delta t_{22}^{1}=-\frac{(q E)^{2}(\beta l)^{6}}{6 s^{4}} I_{3}\left(\frac{(\beta l)^{2}}{s}\right) \\
& \Delta t_{33}^{1}=\frac{(q E)^{2}}{4}\left[\frac{1}{3 s}-\frac{(\beta l)^{4}}{s^{3}} I_{2}\left(\frac{(\beta l)^{2}}{s}\right)\right]+(q E)^{2}(t+T / 2)^{2} \frac{(\beta l)^{4}}{s^{4}} I_{2}\left(\frac{(\beta l)^{2}}{s}\right), \\
& \Delta t_{k k}^{2}=\frac{(q E)^{2}}{6}\left[\frac{1}{2 s}+\frac{(\beta l)^{2}}{4 s^{2}}-\frac{(\beta l)^{6}}{s^{4}} I_{3}\left(\frac{(\beta l)^{2}}{s}\right)\right] \\
& -(q E)^{2}(t+T / 2)^{2} \frac{(\beta l)^{2}}{2 s^{3}}\left[I_{1}\left(\frac{(\beta l)^{2}}{s}\right)-\frac{(\beta l)^{2}}{s} I_{2}\left(\frac{(\beta l)^{2}}{s}\right)\right]
\end{aligned}
$$

where the relation $\Phi_{0}(0)=1$ for the integral (100) has been used, and we have neglected the terms smaller that the remaining ones when $s \sim(\beta l)^{2}$.

Using the representation 9.1(2) in [34,

$$
\Gamma\left(\frac{1}{2}, \frac{p}{4}\right)=\frac{p^{1 / 2}}{2} \int_{1}^{\infty} \frac{d u}{u^{1 / 2}} e^{-u p / 4},
$$

and formula (91), one can calculate the following integrals:

$$
\chi_{n}=\int_{0}^{\infty} s^{-N} \exp \left[-M^{2} s-\frac{(\beta l)^{2}}{4 s}\right] I_{n}\left(\frac{(\beta l)^{2}}{s}\right) d s, \quad N \geq 2 .
$$

Let us first examine the case $n=1$. Calculating the integral over $s$, we obtain

$$
\chi_{1}=\frac{2^{N-1} M^{2 N-3}}{\beta l} \int_{M \beta l}^{\infty} z^{1-N} K_{N-1}(z) d z .
$$

This representation allows one to extract the leading contribution for $M \beta \rightarrow 0$. The function $K_{N-1}(z)$ decreases sufficiently fast at large $z$, and it is easy to see that the leading contribution is formed at the region of small $z, z \leq \delta_{z}$, where $\delta_{z}$ is a small value, such that $M \beta l \ll \delta_{z} \ll 1$. It is sufficient to take into account such $l$ that $M \beta l \ll 1$, since the contributions $\chi_{1}$ into the sum (114) due to the higher values of $l$ can be neglected 6 Neglecting the small contribution given by the region of $z>\delta_{z}$, we now represent $\chi_{1}$ in the form

$$
\chi_{1}=\frac{2^{N-1} M^{2 N-3}}{\beta l} \int_{M \beta l}^{\delta_{z}} z^{1-N} K_{N-1}(z) d z .
$$

This integral can be calculated due to expansion 8.446 [40] for $K_{N-1}(z)$ at $z \ll 1$,

$$
K_{N-1}(z)=\frac{1}{2}\left(\frac{2}{z}\right)^{N-1}(N-2) !
$$

\footnotetext{
${ }^{6}$ As an alternative, one can sum over $l$ the contribution $\chi_{1}$ by using the representation (120) and the summation formula with the function $K_{n}(x l)$ [32. This approach is more effective when one needs to obtain high-temperature expansions beyond the leading terms.
} 
Then, we finally obtain

$$
\chi_{1}=\frac{2^{2 N-1}(N-2) !}{(2 N-3)(\beta l)^{2 N-2}} .
$$

in the leading approximation. Expressing $I_{2}(p)$ through $I_{1}(p)$ by using relation (118) and following the same procedure as in the case of calculating $\chi_{1}$, we obtain the leading term for $\chi_{2}$ at $N \geq 3$, namely,

$$
\chi_{2}=\frac{2^{2 N-3}}{(\beta l)^{2 N-2}}\left[\frac{(N-3) !}{8}+\frac{1}{2(2 N-5)}-\frac{1}{2 N-3}\right] .
$$

In a similar way, we can estimate the leading contribution for $\chi_{3}$. For our purposes, it is sufficient to estimate the orders of $\chi_{3}: \chi_{3} \sim(\beta l)^{-4} \ln (M \beta l)$ at $N=3$, and $\chi_{3} \sim(\beta l)^{-6}$ at $N=4$. Using these results for a calculation of the expressions defined by (116) and (119), and leaving only the leading contributions, we find

$$
\begin{aligned}
& J_{\mu}^{\chi}=\frac{8}{3 \pi^{2}} \frac{j_{\mu}^{0}}{(\beta l)^{2}}, \\
& \Delta T_{11}=\Delta T_{22}=\frac{1}{4 \pi^{2}}\left[\frac{(q E)^{2}}{6} K_{0}(M \beta l)-\frac{58}{15} \frac{(q E)^{2}(t+T / 2)^{2}}{(\beta l)^{2}}\right], \\
& \Delta T_{33}=\frac{1}{4 \pi^{2}}\left[\frac{(q E)^{2}}{3} K_{0}(M \beta l)-\frac{14}{15} \frac{(q E)^{2}(t+T / 2)^{2}}{(\beta l)^{2}}\right] .
\end{aligned}
$$

Proceeding in the same way, we find the leading contribution to $\Delta T_{00}$ as follows:

$$
\Delta T_{00}=\sum_{k=1,2,3} \Delta T_{k k}
$$

Taking the sum of the terms (124), in accordance with the representation (114), we use the alternating zeta-function

$$
\eta(s)=\sum_{l=1}^{\infty}(-1)^{l+1} \frac{1}{l^{s}}
$$

and the summation formula (see, e.g., the Appendix of [32])

$$
\sum_{l=1}^{\infty}(-1)^{l+1} K_{0}(M \beta l)=-\frac{1}{2} \ln (M \beta)+O(1),
$$

in which we have fixed only the leading term, in accordance with our approximation. Since $\eta(2)=\pi^{2} / 12$, we obtain the final result as follows:

$$
\begin{aligned}
& \operatorname{Re}\left\langle j_{\mu}(t)\right\rangle_{\theta}^{c}=-\frac{4}{9} \delta_{\mu}^{3} q^{2} E(t+T / 2) / \beta^{2}, \\
& \operatorname{Re}\left\langle T_{\mu \nu}(t)\right\rangle_{\theta}^{c}=\left.\operatorname{Re}\left\langle T_{\mu \nu}(t)\right\rangle_{\theta}^{c}\right|_{E=0}+\tau_{\mu \nu}^{\theta}(t), \\
& \tau_{11}^{\theta}(t)=\tau_{22}^{\theta}(t)=\frac{(q E)^{2}}{12 \pi^{2}}\left[-\frac{1}{2} \ln (M \beta)+O(1)-\frac{29 \pi^{2}}{15} \frac{(t+T / 2)^{2}}{\beta^{2}}\right], \\
& \tau_{33}^{\theta}(t)=\frac{(q E)^{2}}{12 \pi^{2}}\left[-\ln (M \beta)+O(1)-\frac{7 \pi^{2}}{15} \frac{(t+T / 2)^{2}}{\beta^{2}}\right], \\
& \tau_{00}^{\theta}(t)=\frac{(q E)^{2}}{12 \pi^{2}}\left[-2 \ln (M \beta)+O(1)-\frac{13 \pi^{2}}{3} \frac{(t+T / 2)^{2}}{\beta^{2}}\right] .
\end{aligned}
$$

Even though we examine the case of a small kinetic-momentum increment, the time-dependent terms in $\tau_{\mu \nu}^{\theta}(t)$ (127) may be larger that the logarithmic contributions, because they are limited only by the condition that $(t+T / 2)^{2} / \beta^{2} \ll 1 /\left(q E \beta^{2}\right)^{2}$. The derivative of these terms with respect to time $t$ determines the rate of change for the components $\operatorname{Re}\left\langle T_{\mu \nu}(t)\right\rangle_{\theta}^{c}$.

Let us find the relation of the resulting expression for the current density $\operatorname{Re}\left\langle j_{\mu}(t)\right\rangle_{\theta}^{c}$ with respect to the initial particle density $n^{(\zeta)}$. The leading terms of the effective Lagrangian $\left.\Delta \mathcal{L}_{\theta}\right|_{B=0}$ (92) can be expressed as

$$
\left.\Delta \mathcal{L}_{\theta}\right|_{B=0}=\frac{2}{\pi^{2} \beta^{4}} \sum_{\zeta= \pm}\left[\eta(4)+\eta(3) \beta \mu^{(\zeta)}\right],
$$


where formula (121) has been used. Then, using (88) we obtain

$$
n^{(+)}=n^{(-)}=\frac{2 \eta(3)}{\pi^{2} \beta^{3}},
$$

where $\eta(3)=0,9015426774$, and we have

$$
\operatorname{Re}\left\langle j_{\mu}(t)\right\rangle_{\theta}^{c}=-\frac{\pi^{2}}{9 \eta(3)} \delta_{\mu}^{3} q^{2} E(t+T / 2) \beta \sum_{\zeta= \pm} n^{(\zeta)} .
$$

Let us compare the time-independent terms in (127) with the magnetic field-dependent terms in $\left.\left\langle T_{\mu \nu}(t)\right\rangle_{\theta}^{c}\right|_{E=0}$ (94). Using formula (126), we find the leading field-dependent term of the effective Lagrangian $\Delta \overline{\mathcal{L}}_{\theta}(93)$ at high temperatures:

$$
\mathcal{L}_{1}=-\frac{(q B)^{2}}{12 \pi^{2}}[\ln (M \beta)+O(1)]
$$

which coincides with previously known results (see, e.g., [15]). Then, using formula (94), we find that the magnetic field-dependent terms of EMT are given by

$$
\tau_{00}^{B}=\tau_{11}^{B}=\tau_{22}^{B}=-\tau_{33}^{B}=-\mathcal{L}_{1} .
$$

We can see that the equation of state for the magnetic field in a medium at high temperature is identical with the equation of state for this field in vacuum. The increment of the energy density of magnetic field caused by vacuum polarization, $\left.\left\langle T_{00}(t)\right\rangle_{r e n}^{c}\right|_{E=0}$, and the increment $\tau_{00}^{B}$ are both negative, which holds true in the case of a field of any intensity. On the contrary, the increment of the energy density of electric field caused by vacuum polarization, $\left\langle T_{00}(t)\right\rangle_{\text {ren }}^{c}$, is positive in a weak field and is negative in a strong one. The time-independent terms of $\tau_{\mu \nu}^{\theta}(t)$ (127) are positive. Therefore, even at the initial period of the existence of electric field, when the time-dependent terms of $\tau_{\mu \nu}^{\theta}(t)$ can be neglected, its equation of state is essentially different from the vacuum equation and has different forms in the cases of weak and strong fields.

Large increment of kinetic momentum When the increment of kinetic momentum is large, $|q E|(t+T / 2) \beta \gg$ 1 , the leading contribution to the integral over $u$ in (114) is formed at the region $u \gg 1$. In order to find the asymptotic behavior of expressions (114), it is sufficient to know a rough approximation of the function $Y^{W}$, namely,

$$
Y^{W}=Y_{0}=\exp \left[-(q E)^{2}(t+T / 2)^{2}(\beta l)^{2} / u\right] .
$$

Then $J_{\mu}^{\chi}$ and $T_{\mu \nu}^{\chi}$ can be approximated as follows:

$$
\begin{aligned}
J_{\mu}^{\chi} & =\int_{0}^{\infty} d s \int_{0}^{\infty} d u h^{0} Y_{0} j_{\mu}^{W}, \\
T_{\mu \nu}^{\chi} & =\int_{0}^{\infty} d s \int_{0}^{\infty} d u\left(h^{0}+\delta h\right) Y_{0}\left(t_{\mu \iota}^{0}+\delta t_{\mu \iota}\right),
\end{aligned}
$$

with $t_{\mu \iota}^{0}$ given by (84), and $h^{0}, \delta h, \delta t_{\mu \iota}$ given by (97), where the approximation $\tau_{W}=(\beta l)^{2} / u$ has been used. It is convenient to carry out the integration over $u$ in (129) with the help of the generating function $Y_{0}(\sigma)=\exp \left[-(q E)^{2}(t+T / 2)^{2}(\beta l)^{2} \sigma / u\right], \sigma>0$, which is identical with $Y_{0}$ at $\sigma=1$. Using formula (139), we obtain

$$
\frac{1}{2 \sqrt{\pi}} \int_{0}^{\infty} \frac{d u}{u^{1 / 2}} e^{-u / 4} Y_{0}(\sigma)=\exp \left[-|q E|(t+T / 2) \beta l \sigma^{1 / 2}\right] .
$$

This formula, along with the first and second derivative with respect to the parameter $\sigma$, allows one to carry out the integration over $u$ in (129). As a result, we find, with accuracy up to the pre-exponential factor, that $J_{\mu}^{\chi}$ and $T_{\mu \nu}^{\chi}$ are of the order $\exp [-|q E|(t+T / 2) \beta l]$. Consequently, the leading contribution is given by the terms $J_{\mu}^{\chi}$ and $T_{\mu \nu}^{\chi}$ for $l=1$. Therefore, considering asymptotically large kinetic momenta, one can see that nonzero $\operatorname{Re}\left\langle j_{\mu}(t)\right\rangle_{\theta}^{c}$ and $\operatorname{Re}\left\langle T_{\mu \nu}(t)\right\rangle_{\theta}^{c}$ decrease exponentially:

$$
\begin{aligned}
\operatorname{Re}\left\langle j_{3}(t)\right\rangle_{\theta}^{c} & \sim \exp [-|q E|(t+T / 2) \beta], \\
\operatorname{Re}\left\langle T_{\mu \mu}(t)\right\rangle_{\theta}^{c} & \sim \exp [-|q E|(t+T / 2) \beta] .
\end{aligned}
$$

A calculation of the pre-exponential factor requires a more accurate approximation and some very tedious calculations. 


\subsubsection{Strong electric field}

In the limit of a strong electric field, $|q E| \gg M^{2}, \beta^{-2},|q B|$, the leading contributions depending on the electric field for the integrals $\operatorname{Re} J_{\mu}^{(l)}$ and $\operatorname{Re} T_{\mu \nu}^{(l)}$ in (76) and (77) emerge from large values of $|q E| s$ and $|q E| \tau,|q E| \tau \gg|q E| s \gg 1$. For simplicity, we assume that $B=0$. In addition, we know from Subsec. 3.2 that the leading time-dependent vacuum contributions, $\operatorname{Re}\left\langle j_{\mu}(t)\right\rangle^{p}$ and $\operatorname{Re}\left\langle T_{\mu \nu}(t)\right\rangle^{p}$ do not depend on the details of switching on and off when the time interval $t-t_{1}=t+T / 2$ is sufficiently large, so that condition (38) holds true. Then, it is instructive to examine thermal contributions for the same large interval. In this case, we can use the following approximation:

$$
\begin{aligned}
& \operatorname{Re}\left\langle j_{\mu}(t)\right\rangle_{\theta}^{c}=\sum_{\zeta= \pm} \sum_{l=1}^{\infty}(-1)^{l+1} e^{\beta l \mu^{(\zeta)}} \operatorname{Re} J_{\mu}^{(l)}, \\
& J_{\mu}^{(l)}=j_{\mu}^{0} \int_{0}^{\infty} d s \int_{0}^{\infty} d u h^{s} Y^{s} ;
\end{aligned}
$$

and

$$
\begin{aligned}
& \operatorname{Re}\left\langle T_{\mu \nu}(t)\right\rangle_{\theta}^{c}=\sum_{\zeta= \pm} \sum_{l=1}^{\infty}(-1)^{l+1} e^{\beta l \mu^{(\zeta)}} \operatorname{Re} T_{\mu \nu}^{(l)}, \\
& T_{\mu \nu}^{(l)}=\int_{0}^{\infty} d s \int_{0}^{\infty} d u h^{s} Y^{s} t_{\mu \nu}^{s}, \\
& t_{00}^{s}=\sum_{k=1,2,3} t_{k k}^{s}+M^{2} e^{|q E| s} / 2, t_{11}^{s}=t_{22}^{s}=\frac{u}{2(\beta l)^{2}}, \\
& t_{33}^{s}=2 e^{-|q E| s}\left[\frac{u}{2(\beta l)^{2}}+(q E)^{2}(t+T / 2)^{2}\right]
\end{aligned}
$$

where

$$
\begin{aligned}
h^{s} & =\frac{1}{2} D_{l}|q E| u \exp \left[\frac{i \pi}{4}-\left(|q E|+i M^{2}\right) s-\frac{u}{4}-\frac{(M \beta l)^{2}}{u}\right], \\
D_{l} & =\left[2 \pi^{5 / 2} \sqrt{|q E|}(\beta l)^{3}\right]^{-1} \\
Y^{s} & =\exp \left[-i|q E|(t+T / 2)^{2}-(t+T / 2)^{2}(\beta l)^{-2} u\right]
\end{aligned}
$$

and $j_{\mu}^{0}$ is given by expression (103). In this representation, the kernels are products: a function of the variable $s$ times a function of the variable $u$; therefore, these double integrals can be represented as a product: an integral over $s$ times an integral over $u$. Having calculated the integral over $s$, and using formula (91) for a calculation of the integral over $u$, we find that

$$
\begin{aligned}
\operatorname{Re} J_{\mu}^{(l)} & =2 j_{\mu}^{0} \cos \left[\frac{\pi}{4}-|q E|(t+T / 2)^{2}\right] D_{l} f_{1}, \\
\operatorname{Re} T_{00}^{(l)} & =\operatorname{Re} T_{33}^{(l)}+\sin \left[\frac{\pi}{4}-|q E|(t+T / 2)^{2}\right] D_{l}|q E| f_{1}, \\
\operatorname{Re} T_{11}^{(l)} & =\operatorname{Re} T_{22}^{(l)}=\cos \left[\frac{\pi}{4}-|q E|(t+T / 2)^{2}\right] \frac{D_{l} f_{2}}{(\beta l)^{2}}, \\
\operatorname{Re} T_{33}^{(l)} & =\cos \left[\frac{\pi}{4}-|q E|(t+T / 2)^{2}\right] D_{l}\left[\frac{f_{2}}{(\beta l)^{2}}+2(q E)^{2}(t+T / 2)^{2} f_{1}\right],
\end{aligned}
$$

where

$$
f_{n}=\left(\frac{M \beta l}{\sqrt{1 / 4+(t+T / 2)^{2}(\beta l)^{-2}}}\right)^{n+1} K_{n+1}\left(\sqrt{(M \beta l)^{2}+4 M^{2}(t+T / 2)^{2}}\right) .
$$

We note that the dimensionless parameter $M(t+T / 2)$ is equal to unity for the time period taken by light to cross the Compton radius of a particle of mass $M$. Thus, for a macroscopic period of time, 
$M(t+T / 2) \gg 1$, one can use the asymptotic expansion (105), namely,

$$
\begin{aligned}
& K_{n+1}\left(\sqrt{(M \beta l)^{2}+4 M^{2}(t+T / 2)^{2}}\right) \\
& =\sqrt{\frac{\pi}{2 \sqrt{(M \beta l)^{2}+4 M^{2}(t+T / 2)^{2}}}} \exp \left[-\sqrt{(M \beta l)^{2}+4 M^{2}(t+T / 2)^{2}}\right] .
\end{aligned}
$$

At low initial temperatures, $M \beta \gg 1$, this asymptotic expansion is valid for any period of time. We can see that in the limit of a strong electric field the behavior of the current density $\operatorname{Re}\left\langle j_{\mu}(t)\right\rangle_{\theta}^{c}$ and the EMT components $\operatorname{Re}\left\langle T_{\mu \nu}(t)\right\rangle_{\theta}^{c}$ is described by relaxation oscillations. The amplitudes of these oscillations are small in comparison with the increasing vacuum contributions $\operatorname{Re}\left\langle j_{\mu}(t)\right\rangle^{p}$ and $\operatorname{Re}\left\langle T_{\mu \nu}(t)\right\rangle^{p}$ in (50), respectively, starting from the instant $t$ when conditions (38) are fulfilled.

\section{Discussion and summary}

We have obtained non-perturbative one-loop representations for the mean current density $\left\langle j_{\mu}(t)\right\rangle$ and renormalized EMT $\left\langle T_{\mu \nu}(t)\right\rangle_{r e n}$ of a Dirac field in a constant electric-like background. Two cases of initial states are considered, the vacuum state and a thermal equilibrium state. In the general case, each of the obtained expressions consists of three characteristic terms:

$$
\begin{aligned}
\left\langle j_{\mu}(t)\right\rangle & =\operatorname{Re}\left\langle j_{\mu}(t)\right\rangle_{\theta}^{c}+J_{\mu}^{p}(t), \\
\left\langle T_{\mu \nu}(t)\right\rangle_{r e n} & =\operatorname{Re}\left\langle T_{\mu \nu}(t)\right\rangle_{r e n}^{c}+\operatorname{Re}\left\langle T_{\mu \nu}(t)\right\rangle_{\theta}^{c}+\tau_{\mu \nu}^{p}(t) ;
\end{aligned}
$$

see (65).

The components $\operatorname{Re}\left\langle T_{\mu \nu}(t)\right\rangle_{\text {ren }}^{c}$ given by (59) describe the contribution due to vacuum polarization. The corresponding components of current density are zero, $\left\langle j_{\mu}(t)\right\rangle^{c}=0$. These components $\operatorname{Re}\left\langle T_{\mu \nu}(t)\right\rangle^{c}$ can be related with the real part of Heisenberg-Euler Lagrangian. They are local, i.e., they depend on $t$, but do not depend on the history of the process.

The components $\operatorname{Re}\left\langle j_{\mu}(t)\right\rangle_{\theta}^{c}$ and $\operatorname{Re}\left\langle T_{\mu \nu}(t)\right\rangle_{\theta}^{c}$ given by (76), (77), (79) describe the contribution due to the work of the external field on the particles at the initial state. The components $J_{\mu}^{p}(t)$ and $\tau_{\mu \nu}^{p}(t)$ given by (48), (50), (69) describe the contribution due to the creation of real particles from vacuum. These contributions depend on the time interval $t-t_{1}$ that passes since the instant the electric field turns on, and therefore they are global quantities.

All these contributions are investigated in detail in different regimes, limits of weak and strong fields and low and high temperatures. In all these limiting cases, we have obtained the leading contributions, which are given by elementary functions of the basis dimensionless parameters.

The action of the electric field manifests itself differently for different components of the EMT $\left\langle T_{\mu \nu}(t)\right\rangle_{r e n}$. For instance, in a strong electric field, or in a field which is not strong but acts for a sufficiently long time interval, there is the following correspondence: a coincidence of the leading terms for energy density, $\operatorname{Re}\left\langle T_{00}(t)\right\rangle_{\theta}^{c}+\tau_{00}^{p}(t)$, and the pressure along the direction of the electric field, $\operatorname{Re}\left\langle T_{33}(t)\right\rangle_{\theta}^{c}+\tau_{33}^{p}(t)$. Note that for vacuum polarization the terms in the mentioned correspondence have the opposite signs: $\operatorname{Re}\left\langle T_{00}(t)\right\rangle_{\text {ren }}^{c}=-\operatorname{Re}\left\langle T_{33}(t)\right\rangle_{\text {ren }}^{c}$. This happens because in the former relation we deal with the equation of state for the relativistic fermions, while in the latter relation we deal with the equation of state for the electromagnetic field. The dependence of the electric field and its duration for the transversal component $\operatorname{Re}\left\langle T_{11}(t)\right\rangle_{\theta}^{c}+\tau_{11}^{p}(t)$ is quite different from the dependence of the longitudinal component $\operatorname{Re}\left\langle T_{33}(t)\right\rangle_{\theta}^{c}+\tau_{33}^{p}(t)$. This means that one cannot define a universal functional for the electric field, whose variations should produce all the components of the EMT. This is valid not only in case there exist particles at the initial state, but also when the initial state is vacuum. Therefore, we can see that there cannot be any generalization of the Heisenberg-Euler Lagrangian for the problem of calculating the mean values. In our opinion, this fact takes place for any pair-creating background, and is related, in such a case, to the existence of nonlocal contributions to the EMT. This explains the failure of numerous attempts to examine one-loop effects on the basis of different variants [19, 26] of such a generalization.

Making a comparison of the constant temperature-dependent components of the mean energy density and pressure in a weak electric field immediately after it switches on, we can see that in this case any generalization of the Heisenberg-Euler Lagrangian to the case of finite temperature is impossible. In other words, even a seemingly small disturbance of thermal equilibrium, produced by a weak electric field, exceeds the limits of applicability of the approaches based on thermal equilibrium.

We have established the restriction $|q E| T^{2} \ll \frac{\pi^{2}}{2 q^{2}}$ (which takes place both for the initial vacuum state and for a low-temperature initial thermal state) for the strength and duration of electric field under which QED with a strong constant electric field remains consistent. Under this restriction, one can neglect the back-reaction of particles created by the electric field. On the other hand, there exists another restriction, 
$1 \ll|q E| T^{2}$, see (38), that allows one to disregard the details of switching the electric field on and off. Gathering both restrictions, we obtain the following range of the dimensionless parameter $|q E| T^{2}$ under which QED with a strong constant electric field is consistent:

$$
1 \ll|q E| T^{2} \ll \pi^{2} / 2 q^{2} .
$$

This inequality is consistent due to $\pi^{2} / 2 q^{2} \gg 1$, and one can be certain that QED with a $T$-constant field does exist.

Similar restrictions can be obtained when the initial thermal equilibrium is taken at sufficiently high temperatures, $\beta|q E| T \ll 1$. In this case, we have two inequalities, $\beta|q E|^{2} T^{3} \ll \frac{3 \pi^{2}}{q^{2}}$ and $1 \ll|q E| T^{2}$, which imply

$$
1 \ll|q E| T^{2} \ll \frac{3 \pi^{2}}{q^{2} \beta|q E| T} .
$$

We can see that the upper restriction for $|q E| T^{2}$ is weaker than in the low-temperature case.

We believe that a similar consideration for an electric-like non-Abelian external field will lead to the same restrictions when the created Fermi particles (partons) can be treated as weakly coupled. In the case of high temperatures, one can neglect the back-reaction of the created particles on the chromoelectric field, in comparison with the contribution from the bosons, which are influenced by temperature in a different way (the case of bosons will be examined in another work).

\section{Appendix}

\section{.1 Separation of particle creation contributions}

Using relations (7), we find

$$
\begin{aligned}
\pm \tilde{S}_{\theta}^{\mp}\left(x, x^{\prime}\right) & =S_{\theta, \pm}\left(x, x^{\prime}\right)+S_{\theta, \pm}^{p}\left(x, x^{\prime}\right) \\
S_{\theta,+}\left(x, x^{\prime}\right) & =-i \sum_{n, m} N_{m}^{(+)}(i n)^{+} \psi_{n}(x) G\left(\left.{ }_{+}\right|^{+}\right)_{n m}^{-1}+\bar{\psi}_{m}\left(x^{\prime}\right), \\
S_{\theta,-}\left(x, x^{\prime}\right) & =i \sum_{n, m} N_{n}^{(-)}(i n)-\psi_{n}(x)\left[G\left(\left.{ }_{-}\right|^{-}\right)^{-1}\right]_{n m}^{\dagger}{ }^{-} \bar{\psi}_{m}\left(x^{\prime}\right), \\
S_{\theta,+}^{p}\left(x, x^{\prime}\right) & =-i \sum_{n m} N_{m}^{(+)}(i n)-\psi_{n}(x)\left[G\left(\left.\left.\right|_{+}\right|^{-}\right) G\left(\left.{ }_{-}\right|^{-}\right)^{-1}\right]_{n m}^{\dagger}+\bar{\psi}_{m}\left(x^{\prime}\right), \\
S_{\theta,-}^{p}\left(x, x^{\prime}\right) & =-i \sum_{n m} N_{n}^{(-)}(i n)-\psi_{n}(x)\left[G\left(+\left.\right|^{-}\right) G\left(-\left._{-}\right|^{-}\right)^{-1}\right]_{n m}^{\dagger}+\bar{\psi}_{m}\left(x^{\prime}\right) .
\end{aligned}
$$

Taking into account (9), we can see that $S_{\theta, \pm}^{p}$ describe the contributions due to particle-creation.

Then, we represent the real-valued terms $\left\langle j_{\mu}(t)\right\rangle^{\theta}$ and $\left\langle T_{\mu \nu}(t)\right\rangle^{\theta}$ in (27) as follows:

$$
\begin{gathered}
\left\langle j_{\mu}(t)\right\rangle^{\theta}=\operatorname{Re}\left\langle j_{\mu}(t)\right\rangle_{\theta}^{c}+\operatorname{Re}\left\langle j_{\mu}(t)\right\rangle_{\theta}^{p}, \\
\left\langle T_{\mu \nu}(t)\right\rangle^{\theta}=\operatorname{Re}\left\langle T_{\mu \nu}(t)\right\rangle_{\theta}^{c}+\operatorname{Re}\left\langle T_{\mu \nu}(t)\right\rangle_{\theta}^{p},
\end{gathered}
$$

where

$$
\begin{aligned}
\left\langle j_{\mu}(t)\right\rangle_{\theta}^{c} & =\left.i q \sum_{\zeta= \pm} \operatorname{tr}\left[\gamma_{\mu} S_{\theta, \zeta}\left(x, x^{\prime}\right)\right]\right|_{x=x^{\prime}},\left\langle T_{\mu \nu}(t)\right\rangle_{\theta}^{c}=\left.i \sum_{\zeta= \pm} \operatorname{tr}\left[A_{\mu \nu} S_{\theta, \zeta}\left(x, x^{\prime}\right)\right]\right|_{x=x^{\prime}} \\
\left\langle j_{\mu}(t)\right\rangle_{\theta}^{p} & =\left.i q \sum_{\zeta= \pm} \operatorname{tr}\left[\gamma_{\mu} S_{\theta, \zeta}^{p}\left(x, x^{\prime}\right)\right]\right|_{x=x^{\prime}},\left\langle T_{\mu \nu}(t)\right\rangle_{\theta}^{p}=\left.i \sum_{\zeta= \pm} \operatorname{tr}\left[A_{\mu \nu} S_{\theta, \zeta}^{p}\left(x, x^{\prime}\right)\right]\right|_{x=x^{\prime}}
\end{aligned}
$$

Because of the symmetry $\gamma^{0} \tilde{S}_{\theta}^{\mp}\left(x, x^{\prime}\right)^{\dagger} \gamma^{0}=\tilde{S}_{\theta}^{\mp}\left(x^{\prime}, x\right)$, which can be observed in representation (19), we can calculate the real-valued parts of the right-hand sides of (137) at $x=x^{\prime}$ as follows:

$$
\begin{aligned}
& \left.\operatorname{tr}\left[\gamma_{\mu} S_{\theta,+}\left(x, x^{\prime}\right)\right]\right|_{x=x^{\prime}}=\left.\lim _{x_{0} \rightarrow x_{0}^{\prime}+0} \operatorname{tr}\left[\gamma_{\mu} S_{\theta,+}\left(x, x^{\prime}\right)\right]\right|_{\mathbf{x}=\mathbf{x}^{\prime}}, \\
& \left.\operatorname{tr}\left[\gamma_{\mu} S_{\theta,-}\left(x, x^{\prime}\right)\right]\right|_{x=x^{\prime}}=\left.\lim _{x_{0} \rightarrow x_{0}^{\prime}-0} \operatorname{tr}\left[\gamma_{\mu} S_{\theta,-}\left(x, x^{\prime}\right)\right]\right|_{\mathbf{x}=\mathbf{x}^{\prime}},
\end{aligned}
$$

and so on. 


\section{.2 Proper-time representation of $S_{\theta, \zeta}$}

As has been mentioned, we know the form of the function $\tilde{S}_{\theta}^{\mp}$ in (19) until the moment $t_{1}$ the electric field turns on. Therefore, we continue our analysis in the case of $t>t_{1}, t^{\prime}>t_{1}$, which we have began in the previous section. We are now interested in the components of this functions, which are denoted in (135) as $S_{\theta, \zeta}$.

Because of the cutting-off factor, the integrals $S_{\theta, \zeta}$ are finite. However, they have derivatives proportional to the time interval, $t-t_{1}$, which may be large. Therefore, in the study of conventional contributions we have to pay attention to the contributions that grow as $t-t_{1}$ increases. Note that, as distinct from the contributions $J_{\mu}^{p}(t)$ and $\tau_{\mu \nu}^{p}(t)$, which have been estimated only for sufficiently large intervals $t-t_{1}$, conventional contributions are calculated for any $t, 0 \leq t-t_{1} \leq T$.

We remind that the functions $\zeta \psi_{n}(x)$ and ${ }^{\zeta} \psi_{n}(x)$ are solutions of the Dirac equation in a constant field, described in Subsec. 3.1. In the relation (35) of that subsection, we have presented the expressions for the coefficients $G\left(-\left.\right|^{+}\right)$, obtained for the $T$-constant field, which determine all the coefficients $G\left(\left.\zeta_{\zeta}\right|^{\zeta}\right)$ via the unitarity condition. These expressions for $G\left(-\left.\right|^{+}\right)$at longitudinal momenta $p_{3}$ being large and comparable with $|q E| T$ are different from the asymptotic form at $T \rightarrow \infty$. Accordingly, for such large values $p_{3}$ the coefficients $G\left(\left.\zeta\right|^{\zeta}\right)$ are different from the corresponding asymptotic forms. However, it can be shown that under condition (75) these differences in expressions for $S_{\theta, \zeta}$ can be neglected, and one can use, for every $p_{3}$, the asymptotic form of the coefficients $G\left(\zeta^{\zeta}\right)$, which holds true for a constant field. On the other hand, we need to attract attention to the above-mentioned fact, which will be used to obtain the necessary representation, whereas a manifest form of these coefficients in a constant field will be unnecessary.

The energy spectrum of $i n$-particles at $t<t_{1}$ has the form

$$
\varepsilon_{n}^{(\zeta)}=\varepsilon_{n}=\sqrt{M^{2}+\omega+\left(\pi_{3}\right)^{2}}, \pi_{3}=\left(\frac{q E T}{2}+p_{3}\right),
$$

where

$$
\begin{aligned}
& n=\left(p_{1}, n_{B}, p_{3}, r\right), \omega=|q B|\left(2 n_{B}+1-r\right), n_{B}=0,1, \ldots, B \neq 0 \\
& n=(\mathbf{p}, r), \omega=p_{1}^{2}+p_{2}^{2}, \quad B=0 .
\end{aligned}
$$

We expand $N_{n}^{(\zeta)}($ in $)(20)$ as a power series of $\exp \left\{-\beta\left(\varepsilon_{n}^{(\zeta)}-\mu^{(\zeta)}\right)\right\}$ and make use of the formula (see [40, Eqs. 3.471.9 and 8.469.3)

$$
\exp \left\{-\beta l \varepsilon_{n}\right\}=\frac{1}{2 \sqrt{\pi}} \int_{0}^{\infty} \frac{d u}{u^{1 / 2}} \exp \left(-\frac{u}{4}-\frac{\beta^{2} l^{2} \varepsilon_{n}^{2}}{u}\right) .
$$

Since the operator $\left(\mathcal{H}\left(t_{1}\right)\right)^{2}=M^{2}-\left(\boldsymbol{\gamma} \mathbf{P}_{\perp}\right)^{2}+\left(i \partial_{3}+q E T / 2\right)^{2}$ is an integral of motion in the $T$-constant field, the relation

$$
\left(\mathcal{H}\left(t_{1}\right)\right)^{2}{ }_{ \pm} \psi_{n}(x)=\varepsilon_{n \pm}^{2} \psi_{n}(x)
$$

holds true at any time instant $t$. Then, $S_{\theta, \zeta}(135)$ can be represented as follows:

$$
\begin{aligned}
S_{\theta,+}\left(x, x^{\prime}\right) & =i \sum_{n, m} \Upsilon^{(+)+} \psi_{n}(x) G\left(\left.{ }_{+}\right|^{+}\right)_{n m}^{-1}+\bar{\psi}_{m}\left(x^{\prime}\right), \\
S_{\theta,-}\left(x, x^{\prime}\right) & =-i \sum_{n, m} \Upsilon^{(-)}{ }_{-} \psi_{n}(x)\left[G\left({ }_{-}^{-}\right)^{-1}\right]_{n m}^{\dagger}-\bar{\psi}_{m}\left(x^{\prime}\right),
\end{aligned}
$$

where

$$
\begin{aligned}
\Upsilon^{(\zeta)} & =\sum_{l=1}^{\infty}(-1)^{l} e^{\beta l \mu^{(\zeta)}} \frac{1}{2 \sqrt{\pi}} \int_{0}^{\infty} \frac{d u}{u^{1 / 2}} \exp \left(-\frac{u}{4}-\frac{\beta^{2} l^{2} \mathcal{E}_{\zeta}^{2}}{u}\right) \\
\mathcal{E}_{+}^{2} & =M^{2}-\left(\boldsymbol{\gamma} \mathbf{P}_{\perp}^{*}\right)^{2}+\left(-i \partial_{3}^{\prime}+q E T / 2\right)^{2} \\
\mathcal{E}_{-}^{2} & =M^{2}-\left(\boldsymbol{\gamma} \mathbf{P}_{\perp}\right)^{2}+\left(i \partial_{3}+q E T / 2\right)^{2} .
\end{aligned}
$$

Representations (140) and (22) are formally related as follows:

$$
S_{\theta, \pm}\left(x, x^{\prime}\right)= \pm \Upsilon^{( \pm)} S^{\mp}\left(x, x^{\prime}\right) .
$$


To calculate $\left\langle j_{\mu}(t)\right\rangle_{\theta}^{c}$ and $\left\langle T_{\mu \nu}(t)\right\rangle_{\theta}^{c}$ in (137) according to the prescription (138), we need the expressions for $S_{\theta,+}\left(x, x^{\prime}\right)$ at $x_{0}>x_{0}^{\prime}$ and $S_{\theta,-}\left(x, x^{\prime}\right)$ at $x_{0}<x_{0}^{\prime}$ only. Under this condition, the proper-time representation for $S^{\mp}\left(x, x^{\prime}\right)$ has the form

$$
\begin{aligned}
S^{\mp}\left(x, x^{\prime}\right) & =(\gamma P+M) \Delta^{\mp}\left(x, x^{\prime}\right), \\
\pm \Delta^{\mp}\left(x, x^{\prime}\right) & =\Delta^{c}\left(x, x^{\prime}\right), x_{0}-x_{0}^{\prime} \gtrless 0,
\end{aligned}
$$

where $\Delta^{c}\left(x, x^{\prime}\right)$ is defined by the proper-time representations (55) and (56); see [1, 33, 36]. For the function $S_{\theta, \zeta}\left(x, x^{\prime}\right)$, we find

$$
\begin{aligned}
S_{\theta, \zeta}\left(x, x^{\prime}\right) & =(\gamma P+M) \Delta_{\theta, \zeta}^{c}\left(x, x^{\prime}\right) \\
\Delta_{\theta, \zeta}^{c}\left(x, x^{\prime}\right) & =\int_{0}^{\infty} f_{\theta, \zeta}\left(x, x^{\prime}, s\right) d s, \quad f_{\theta, \zeta}\left(x, x^{\prime}, s\right)=\Upsilon^{(\zeta)} f\left(x, x^{\prime}, s\right),
\end{aligned}
$$

where $f\left(x, x^{\prime}, s\right)$ is given by (56).

Thus, we also have proper-time representations for the quantities $\left\langle j_{\mu}(t)\right\rangle_{\theta}^{c}$ and $\left\langle T_{i k}(t)\right\rangle_{\theta}^{c}$. The latter quantities are finite at finite $t-t_{1}$. This implies that representation (143) can be used in calculating the quantities $\left\langle j_{\mu}(t)\right\rangle_{\theta}^{c}$ and $\left\langle T_{i k}(t)\right\rangle_{\theta}^{c}$. Then, the quantity $\left\langle T_{00}(t)\right\rangle_{\theta}^{c}$ must be calculated via $\left\langle T_{i k}(t)\right\rangle_{\theta}^{c}$ as follows:

$$
\left\langle T_{00}(t)\right\rangle_{\theta}^{c}=\sum_{k=1,2,3}\left\langle T_{k k}(t)\right\rangle_{\theta}^{c}+\left.i M \sum_{\zeta= \pm} \operatorname{tr} S_{\theta, \zeta}\left(x, x^{\prime}\right)\right|_{x=x^{\prime}},
$$

The latter follows from (137), with allowance for the fact that $S_{\theta, \zeta}$ are solutions of the Dirac equation. Then, we represent expressions in (137) and (144) in the form

$$
\begin{aligned}
\left\langle j_{\mu}(t)\right\rangle_{\theta}^{c} & =\left.i q \sum_{\zeta= \pm} \operatorname{tr}\left[\gamma_{\mu} \gamma P \Delta_{\theta, \zeta}^{c}\left(x, x^{\prime}\right)\right]\right|_{x=x^{\prime}}, \\
\left\langle T_{\mu \nu}(t)\right\rangle_{\theta}^{c} & =\left.i \sum_{\zeta= \pm} \operatorname{tr}\left[A_{\mu \nu} \gamma P \Delta_{\theta, \zeta}^{c}\left(x, x^{\prime}\right)\right]\right|_{x=x^{\prime}}, \quad \mu, \nu \neq 0 \\
\left\langle T_{00}(t)\right\rangle_{\theta}^{c} & =\sum_{k=1,2,3}\left\langle T_{k k}(t)\right\rangle_{\theta}^{c}+\left.i M^{2} \sum_{\zeta= \pm} \operatorname{tr}\left[\Delta_{\theta, \zeta}^{c}\left(x, x^{\prime}\right)\right]\right|_{x=x^{\prime}} .
\end{aligned}
$$

Following Schwinger, see [8], the kernel $f\left(x, x^{\prime}, s\right)$ can be treated as a matrix element of an operator in the $x$-representation:

$$
f\left(x, x^{\prime}, s\right)=i\left\langle x\left|\exp \left\{-i s\left[M^{2}-(\gamma \hat{P})^{2}\right]\right\}\right| x^{\prime}\right\rangle \text {. }
$$

Here, $\left|x^{\prime}\right\rangle$ is a complete set of eigenvectors of some commuting operators $\hat{X}^{\mu}$, such that $\hat{X}^{\mu}|x\rangle=x^{\mu}|x\rangle$, $\left\langle x \mid x^{\prime}\right\rangle=\delta^{(4)}\left(x-x^{\prime}\right)$. There exist canonically conjugate operators $\hat{P}_{\mu}$ and the commutation relations $\left[\hat{X}^{\mu}, \hat{P}_{\nu}\right]=i \delta_{\nu}^{\mu},\left[\hat{P}_{\nu}, \hat{P}_{\nu}\right]=-i q F_{\mu \nu}$, where $F_{\mu \nu}$ is the field strength of the external field under consideration. Using the representation (146), we rewrite $f_{\theta, \zeta}\left(x, x^{\prime}, s\right)$ (143) as follows:

$$
f_{\theta, \zeta}\left(x, x^{\prime}, s\right)=\sum_{l=1}^{\infty}(-1)^{l} e^{\beta l \mu^{(\zeta)}} \frac{1}{2 \sqrt{\pi}} \int_{0}^{\infty} \frac{d u}{u^{1 / 2}} e^{-u / 4} g\left(x, x^{\prime}, s, \tau\right),
$$

where

$$
\begin{aligned}
g\left(x, x^{\prime}, s, \tau\right) & =\exp \left[-\tau\left(i \partial_{3}+q E T / 2\right)^{2}\right] f\left(x, x^{\prime}, s, \tau\right), \quad \tau=(\beta l)^{2} / u, \\
f\left(x, x^{\prime}, s, \tau\right) & =i \exp \left\{-\tau\left[M^{2}-\left(\gamma \hat{P}_{\perp}\right)^{2}\right]\right\}\left\langle x\left|\exp \left\{-i s\left[M^{2}-(\gamma \hat{P})^{2}\right]\right\}\right| x^{\prime}\right\rangle
\end{aligned}
$$

and

$$
(\gamma \hat{P})^{2}=\left(\gamma \hat{P}_{\|}\right)^{2}+\left(\gamma \hat{P}_{\perp}\right)^{2}, \hat{P}_{\perp}=\left(0, \hat{P}_{1}, \hat{P}_{2}, 0\right), \hat{P}_{\|}=\left(\hat{P}_{0}, 0,0, \hat{P}_{3}\right) .
$$

Since the operators $\left(\gamma \hat{P}_{\|}\right)^{2}$ and $\left(\gamma \hat{P}_{\perp}\right)^{2}$ commute, we present $f\left(x, x^{\prime}, s, \tau\right)$ as follows

$$
f\left(x, x^{\prime}, s, \tau\right)=i\left\langle x\left|\exp \left\{i s\left(\gamma \hat{P}_{\|}\right)^{2}-i(s-i \tau)\left[M^{2}-\left(\gamma \hat{P}_{\perp}\right)^{2}\right]\right\}\right| x^{\prime}\right\rangle .
$$


Applying the Schwinger operator techniques 8 to this expression, we find

$$
\begin{aligned}
& f\left(x, x^{\prime}, s, \tau\right)=f_{\|}\left(x_{\|}, x_{\|}^{\prime}, s\right) f_{\perp}\left(\mathbf{x}_{\perp}, \mathbf{x}_{\perp}^{\prime}, s-i \tau\right), \\
& f_{\|}\left(x_{\|}, x_{\|}^{\prime}, s\right)=\exp \left[i q \Lambda_{\|}+\frac{i}{4}\left(x_{3}-x_{3}^{\prime}\right)^{2} q E \operatorname{coth}(q E s)\right] f_{0}\left(x_{0}, x_{0}^{\prime}, s\right), \\
& f_{0}\left(x_{0}, x_{0}^{\prime}, s\right)=\frac{i q E}{4 \pi \sinh (q E s)} \exp \left[q E s \gamma^{0} \gamma^{3}-\frac{i}{4}\left(x_{0}-x_{0}^{\prime}\right)^{2} q E \operatorname{coth}(q E s)\right], \\
& f_{\perp}\left(\mathbf{x}_{\perp}, \mathbf{x}_{\perp}^{\prime}, s-i \tau\right)=\frac{-i q B}{4 \pi \sin [q B(s-i \tau)]} \exp \left[i q \Lambda_{\perp}-i(s-i \tau)\left(M^{2}-q B \Sigma^{3}\right)+\frac{i q B\left(\mathbf{x}_{\perp}-\mathbf{x}_{\perp}^{\prime}\right)^{2}}{4 \tan [q B(s-i \tau)]}\right], \\
& i q \Lambda_{\perp}=-i \frac{q B}{2}\left(x_{1}-x_{1}^{\prime}\right)\left(x_{2}+x_{2}^{\prime}\right) .
\end{aligned}
$$

The action of the exponential operator in (148) is determined by the expression

$$
\varphi(s, \tau)=\exp \left[-\tau\left(i \partial_{3}+q E T / 2\right)^{2}\right] \exp \left[i q \Lambda_{\|}+\frac{i}{4}\left(x_{3}-x_{3}^{\prime}\right)^{2} q E \operatorname{coth}(q E s)\right] .
$$

Using the integral representation

$$
\int_{-\infty}^{+\infty} e^{-i p^{2} a+i b p} d p=\left(e^{-i \pi / 2} \pi a^{-1}\right)^{1 / 2} e^{i b^{2} / 4 a}
$$

we find

$$
\begin{aligned}
\varphi(s, \tau) & =\left(\frac{a}{a-i \tau}\right)^{1 / 2} \exp \left\{i q \Lambda_{\|}-\left(x_{0}+x_{0}^{\prime}+T\right)^{2}\left(\frac{q E}{2}\right)^{2} \frac{\tau a}{a-i \tau}\right. \\
& \left.+i \frac{b^{2}+i 2 b \tau q E\left(x_{0}+x_{0}^{\prime}+T\right)}{4(a-i \tau)}\right\}
\end{aligned}
$$

where

$$
a=a(s)=(q E)^{-1} \tanh (q E s), b=x_{3}-x_{3}^{\prime} .
$$

Therefore,

$$
g\left(x, x^{\prime}, s, \tau\right)=\varphi(s, \tau) f_{0}\left(x_{0}, x_{0}^{\prime}, s\right) f_{\perp}\left(x_{\perp}, x_{\perp}^{\prime}, s-i \tau\right) .
$$

\section{.3 A convenient representation for weak-field approximation}

First of all, we note that at finite temperatures the kernels in (76) and (77) have singularities due to the zeros of $(a-i \tau)$. If we introduce a new variable $s^{\prime}$,

$$
s^{\prime}=i s+c_{0}, c_{0}=(q E)^{-1} \arctan (q E \tau),
$$

then

$$
(a-i \tau)^{-1}=\frac{q E\left[1+\tan \left(q E s^{\prime}\right) q E \tau\right]}{\tan \left(q E s^{\prime}\right)\left[1+(q E \tau)^{2}\right]} .
$$

The singular points of $(a-i \tau)^{-1}$ are $s^{\prime}= \pm \pi k, k=0,1,2, \ldots$ Let us deform the integration contour over $s$ in integrals (76) and (77) so that it leaves the origin and proceeds downwards along the imaginary axis until the point $-i c_{1}, c_{1}=\left(\pi / 2-c_{0}\right)|q E|^{-1}$, without touching it, and then proceeds in parallel to the axis Re $s$ towards the positive infinity. Then, expressions (76) and (77) can be reorganized as follows:

$$
\begin{aligned}
J_{\mu}^{(l)} & =J_{\mu}^{a}+J_{\mu}^{b}, \\
J_{\mu}^{a} & =-\left.i \int_{0}^{c_{1}-0} d \tilde{s} \int_{0}^{\infty} d u\left[h(s, u) Y(s, u) j_{\mu}(s, u)\right]\right|_{s=-i \tilde{s}}, \\
J_{\mu}^{b} & =\left.\int_{0}^{\infty} d \tilde{s} \int_{0}^{\infty} d u\left[h(s, u) Y(s, u) j_{\mu}(s, u)\right]\right|_{s=\tilde{s}-i\left(c_{1}-0\right)},
\end{aligned}
$$


and

$$
\begin{aligned}
T_{\mu \nu}^{(l)} & =T_{\mu \nu}^{a}+T_{\mu \nu}^{b} \\
T_{\mu \nu}^{a} & =-\left.i \int_{0}^{c_{1}-0} d \tilde{s} \int_{0}^{\infty} d u\left[h(s, u) Y(s, u) t_{\mu \nu}(s, u)\right]\right|_{s=-i \tilde{s}} \\
T_{\mu \nu}^{b} & =\left.\int_{0}^{\infty} d \tilde{s} \int_{0}^{\infty} d u\left[h(s, u) Y(s, u) t_{\mu \nu}(s, u)\right]\right|_{s=\tilde{s}-i\left(c_{1}-0\right)}
\end{aligned}
$$

One can verify that the quantities

$$
\begin{aligned}
\delta J_{\mu}^{a} & =-\left.i \int_{0}^{\infty} d u \int_{e^{i \pi}\left(c_{0}-0\right)}^{0} d \tilde{s}\left[h(s, u) Y(s, u) j_{\mu}(s, u)\right]\right|_{s=-i \tilde{s}}, \\
\delta T_{\mu \nu}^{a} & =-\left.i \int_{0}^{\infty} d u \int_{e^{i \pi}\left(c_{0}-0\right)}^{0} d \tilde{s}\left[h(s, u) Y(s, u) t_{\mu \nu}(s, u)\right]\right|_{s=-i \tilde{s}},
\end{aligned}
$$

are imaginary; therefore the real-valued parts of $J_{\mu}^{a}$ and $T_{\mu \nu}^{a}$ can be represented as

$$
\begin{aligned}
& \operatorname{Re} J_{\mu}^{a}=\operatorname{Re} J_{\mu}^{\prime a}, \quad \operatorname{Re} T_{\mu \nu}^{a}=\operatorname{Re} T_{\mu \nu}^{a}, \\
& J_{\mu}^{a}=J_{\mu}^{a}+\delta J_{\mu}^{a}, \quad T_{\mu \nu}^{\prime a}=T_{\mu \nu}^{a}+\delta T_{\mu \nu}^{a} .
\end{aligned}
$$

We now change the variable $\tilde{s}$ by $s^{\prime}=\tilde{s}+c_{0}$ in the integrals for $J_{\mu}^{\prime a}$ and $T_{\mu \nu}^{\prime a}$. Then, one can see that the contributions from the integration region $0 \leq u<u_{0}$ over $u$ in these integrals are imaginary. Then, changing the variable $u$ by $u^{\prime}$,

$$
u^{\prime}=u-u_{0}, \quad u_{0}=q E(\beta l)^{2} \cot \left(q E s^{\prime}\right),
$$

and introducing the notation $\operatorname{Re} J_{\mu}^{\prime a}=\tilde{J}_{\mu}^{a}, \operatorname{Re} T_{\mu \nu}^{\prime a}=\tilde{T}_{\mu \nu}^{a}$, we represent $\tilde{J}_{\mu}^{a}$ and $\tilde{T}_{\mu \nu}^{a}$ as (80).

Acknowledgement S.P.G. thanks FAPESP for support and Universidade de São Paulo for hospitality. D.M.G. acknowledges FAPESP and CNPq for permanent support.

\section{References}

[1] A.I. Nikishov, in Quantum Electrodynamics of Phenomena in Intense Fields, Proc. P.N. Lebedev Phys. Inst. 111, 153 (Nauka, Moscow 1979).

[2] A.A. Grib, S.G. Mamaev, and V.M. Mostepanenko, Vacuum Quantum Effects in Strong Fields (Atomizdat, Moscow 1988; Friedmann Laboratory Publishing, St. Petersburg 1994).

[3] E.S. Fradkin, D.M. Gitman and S.M. Shvartsman, Quantum Electrodynamics with Unstable Vacuum (Springer-Verlag, Berlin 1991).

[4] W. Greiner, B. Müller, and J. Rafelski, Quantum Electrodynamics of Strong Fields (Springer-Verlag, Berlin 1985); W. Greiner and J. Reinhardt, Quantum Electrodynamics (Springer-Verlag, Berlin 1994).

[5] G.V. Dunne, and C. Schubert, Phys.Rev. D 72, 105004 (2005) arXiv:hep-th/0507174; G.V. Dunne, H. Gies, C. Schubert, and Q.-h. Wang, Phys.Rev. D 73, 065028 (2006) arXiv:hep-th/0602176; G.V. Dunne and Q.-h. Wang, Phys.Rev. D 74, 065015 (2006) arXiv:hep-th/0608020; D.D. Dietrich and G.V. Dunne, arXiv:0706.4006

[6] Y. Kluger, J.M. Eisenberg, and B. Svetitsky, Int. J. Mod. Phys. E 2, 333 (1993); F. Cooper, J.M. Eisenberg, Y. Kluger, E. Mottola, and B. Svetitsky, Phys. Rev. D 48, 190 (1993); J.C.R. Bloch et al, Phys. Rev. D 60, 116011 (1999); D.V. Vinnik et al, Eur. Phys. J. C 22, 341 (2001) arXiv:nucl-th/0103073.

[7] W. Heisenberg and H. Euler, Z. Phys. 98, 714 (1936); English translation available at physics $/ 0605038$.

[8] J. Schwinger, Phys. Rev. 82, 664 (1951).

[9] G.V. Dunne, Heisenberg-Euler effective Lagrangians: Basics and extensions, in Ian Kogan Memorial Volume, From fields to strings: Circumnavigating theoretical physics, Eds. M Shifman, A. Vainshtein and J. Wheater, World Scientific, 2005 arXiv:hep-th/0406216]. 
[10] A. Ringwald, Phys. Lett. B 510, 107 (2001) arXiv:hep-ph/0103185]; Fundamental physics at an X-ray free electron laser, in Proceedings of Erice Workshop on Electromagnetic Probes of Fundamental Physics, W. Marciano and S. White (Eds.). (World Scientific, Singapore, 2003) arXiv:hep-ph/0112254; Boiling the Vacuum with an X-ray Free Electron Laser, in Workshop on Quantum Aspects of Beam Physics, P. Chen and K. Reil (Eds.). (World Scientific, Singapore, 2004) arXiv:hep-ph/0304139 ; R. Alkofer, M.B. Hecht, C.D. Roberts, S.M. Schmidt, and D.V. Vinnik., Phys. Rev. Lett. 87, 193902 (2001); V.S. Popov, JETP Lett. 74, 133 (2001); I.M. Dremin, JETP Lett. 76, 185 (2001).

[11] V.V. Usov, Phys. Rev. Lett. 80, 230 (1998); V.V. Usov, Astrophys. J. Lett. 550, L179 (2001); A.G. Aksenov, M. Milgrom, and V.V. Usov, Mon. Not. R. Astron. Soc. 343, L69 (2003); D. Page and V.V. Usov, Phys. Rev. Lett. 89, 131101(2002); V.V. Usov, Phys. Rev. D 70, 067301 (2004); V.V. Usov, T. Harko, and K.S. Cheng, Astrophys. J. 620, 915 (2005); T. Harko and K.S. Cheng, Astrophys. J. 643, 318 (2006).

[12] J.R.S. Nascimento, I. Cho, A. Vilenkin, Phys. Rev. D 60, 083505 (1999) arXiv:hep-th/9902135.

[13] N. Seiberg, L. Susskind and N. Toumbas, JHEP 0006, 021 (2000); R. Gopakumar, J. Maldacena, S. Minwalla and A. Strominger, JHEP 0006, 036 (2000); P. Mukhopadhyay and A. Sen, JHEP 0211, 047 (2002).

[14] W. Dittrich, Phys. Rev. D 19 (1979), 2385; W. Dittrich and M Reuter, Effective Lagrangians in quantum electrodynamics, Lecture Notes in Physics 220 (Springer-Verlag, Berlin 1985).

[15] D. Cangemi and G. Dunne, Ann. Phys. 249, 582 (1996).

[16] P. Elmfors, D. Persson, and B-S. Skagerstam, Phys. Rev. Lett. 71, 480 (1993); Astroparticle Phys. 2, 299 (1994).

[17] A. Casher, H. Neuberger, and S. Nussinov, Phys. Rev. D 20, 179 (1979); E.G. Gurvich, Phys. Lett. B 87, 386 (1979).

[18] A. Bhattacharyya et al, Nucl. Phys. A 661, 629 (1999); P. Bhattacharyya, J. Alam, B. Sinha, and S. Raha, Phys. Rev. D 48, 4630 (1993).

[19] A.K. Ganguly, P.K. Kaw, and J.C. Parikh, Phys. Rev. C 51, 2091 (1995).

[20] Y. Kluger, E. Mottola, and J.M. Eisenberg, Phys. Rev. D 58, 125015 (1998).

[21] S.M. Schmidt et al., Int. J. Mod. Phys. E 7, 709 (1998); R.S. Bhalerao and G.C. Nayak, Phys. Rev. C 61, 054907 (2000).

[22] S.P. Gavrilov, D.M. Gitman, and J.L. Tomazelli, arXiv:hep-th/0612064; Nucl. Phys. B 795, 645 (2008).

[23] S.P. Gavrilov, D.M. Gitman, and E.S. Fradkin, Yad. Fiz. 46172 (1987) [English. transl.: Sov. J. Nucl. Phys. (USA) 46, 107 (1987)]; in Quantum field theory with unstable vacuum. Proc. P.N. Lebedev Phys. Inst. Vol. 193 (Nauka, Moscow 1989) 208.

[24] I.L. Bukhbinder, D.M. Gitman, and V.P. Frolov, Izv. Vuzov Fizika 23, N.6, 77 (1980) [English. transl.: Sov. Phys. J. 23, 529 (1980)].

[25] J. Hallin and P. Liljenberg, Phys. Rev. D 52, 1150 (1995).

[26] P.H. Cox, W.S. Hellman, and A. Yildiz, Ann. Phys. (N.Y) 154, 211 (1984); M. Loewe and J.C. Rojas, Phys. Rev. D 46, 2689 (1992); P. Elmfors and B.-S. Skagerstam, Phys. Lett. B 348, 141 (1995); 376, 330(E) (1996); A.K. Ganguly, arXiv:hep-th/9804134; H. Gies, Phys. Rev. D 60, 105002 (1999).

[27] V.G. Bagrov, D.M. Gitman, and Sh.M. Shvartsman, Sov. Phys. JETP 41, 191 (1975).

[28] S.P. Gavrilov and D.M. Gitman, Phys. Rev. D 53, 7162 (1996).

[29] D.M. Gitman, J. Phys. A 10, 2007 (1977).

[30] E.S. Fradkin and D.M. Gitman, Fortschr. Phys. 29, 381 (1981).

[31] J. Schwinger, J.Math.Phys. 2, 407 (1961); L.V. Keldysh, Sov. Phys. JETP 20, 1018 (1964).

[32] N.P. Landsman and Ch.G. van Weert, Phys. Rep. 145, 141 (1987). 
[33] A.I. Nikishov, Zh. Eksp. Teor. Fiz. 57, 1210 (1969) [Sov. Phys. JETP 30, 660 (1970)].

[34] Higher Transcendental functions (Bateman Manuscript Project), edited by A. Erdelyi et al. (McGraw-Hill, New York 1953), Vol. 2.

[35] N.B. Narozhny and A.I. Nikishov, Sov. J. Nucl. Phys. (USA) 11, 596 (1970).

[36] S.P. Gavrilov, D.M. Gitman and Sh.M. Shvartsman, Sov. J. Nucl. Phys. (USA) 29, 567 (1979); 715 (1979); S.P. Gavrilov, D.M. Gitman and A.E. Gonçalves, J. Math. Phys. 39, 3547 (1998).

[37] V. Fock, Phys. Z. Sowjetunion 12, 404 (1937).

[38] M. Greenman and F. Rohrlich, Phys. Rev. D 8, 1103 (1973).

[39] V. I. Ritus, The Lagrangian function of an intense electromagnetic field and quantum electrodynamics at short distances, in Issues in Intense-field Quantum Electrodynamics, Proc. P.N. Lebedev Phys. Inst. 168 (Nova Science Pub., New York 1987); arXiv:hep-th/9812124.

[40] I.S. Gradshtein and I.M. Ryzhik Tables of Integrals, Sums, Series and Products (Nauka, Moscow 1971).

[41] J. Kapusta, Finite-temperature field theories (Cambridge university press, Cambridge 1989); M. le Bellac Thermal field theory (Cambridge university press, Cambridge 1996).

[42] A. Actor, Nucl. Phys. B 256, 689 (1986) . 